\title{
A Moist Conceptual Model for the Boundary Layer Structure and Radiatively Driven Shallow Circulations in the Trades
}

\author{
Ann Kristin Naumann, BJorn Stevens, and Cathy Hohenegger \\ Max Planck Institute for Meteorology, Hamburg, Germany
}

(Manuscript received 1 August 2018, in final form 18 December 2018)

\begin{abstract}
A conceptual model is developed to analyze how radiative cooling and the effect of moisture and shallow convection modify the boundary layer (BL) structure and the strength of mesoscale shallow circulations. The moist BL allows for a convective mass flux to modify the BL mass balance, which enhances inversion entrainment compared to a dry case and acts as a moisture valve to the BL. The convective mass flux is found to be insensitive to the applied radiative cooling and in the absence of heterogeneities cloud-free conditions exist only for unusual large-scale forcings. The model is able to explain the moderate range of BL heights and humidities observed in the trades. In a two-column setup, differential radiative BL cooling causes a pressure difference, which drives a BL flow from the cold and moist column to the warm and dry column and couples them dynamically. The small inversion buoyancy jump of the moist BL yields a stronger BL flow of $4 \mathrm{~m} \mathrm{~s}^{-1}$ instead of $1 \mathrm{~m} \mathrm{~s}^{-1}$ in the dry case. For typical conditions of the subsidence-dominated tropical oceans, a radiatively driven shallow circulation is stronger than one driven by sea surface temperature (SST) gradients. While the strength of the SSTdriven circulation decreases with decreasing SST difference, the radiatively driven circulation is insensitive to the radiative BL cooling difference. In both cases, convection is suppressed in the descending branch of the shallow circulation and enhanced in the ascending branch, resembling patterns of organized shallow convection.
\end{abstract}

\section{Introduction}

In regions of large-scale subsidence over the tropical oceans, such as the trades, shallow convection is ubiquitous. These shallow clouds vary little in their cloudbase height and rain frequently although not heavily (e.g., Riehl et al. 1951; Nuijens et al. 2014). There is growing evidence that shallow cumulus clouds are often accompanied by shallow circulations, which are confined to the lower $2-3 \mathrm{~km}$ of the troposphere but are larger than the individual cloud scale and thought to enhance the organization of shallow clouds into patches, clusters, or bands (Bony et al. 2017; Bretherton and Blossey 2017). In this study, we derive a simple conceptual model to assess how the boundary layer (BL) structure is modified by the presence of moist convection, shallow circulations, and their interaction. The derived model shows that a shallow circulation driven by radiative differences can suppress convection in its

\footnotetext{
Corresponding author: Ann Kristin Naumann, ann-kristin.naumann@ mpimet.mpg.de
}

descending branch and enhance convection in its neighboring ascending branch, resembling patterns of organized convection.

The trade wind cumulus regime, which is dominated by shallow clouds and large-scale subsidence, shows moderate subsidence throughout most of the troposphere (Figs. 1a,b; see caption for method and data origin). In regions of low liquid water path (LWP), subsidence increases with height above the surface, maximizing near $850 \mathrm{hPa}$. In regions of high LWP mean ascending motion prevails below $800 \mathrm{hPa}$ and is associated with active shallow convection and higher low-level cloud fraction. This vertical velocity pattern is associated with horizontal convergence at $800 \mathrm{hPa}$ in lowLWP regions and divergence at $800 \mathrm{hPa}$ in high-LWP regions. At the surface, regions of low LWP are dominated by divergence and regions of high LWP are dominated by convergence. Together these patterns depict a shallow mesoscale circulation with increasing low-level subsidence in regions of low convective activity, mean ascending motion in regions of active shallow convection, a shallow outflow from convective to 
(a)

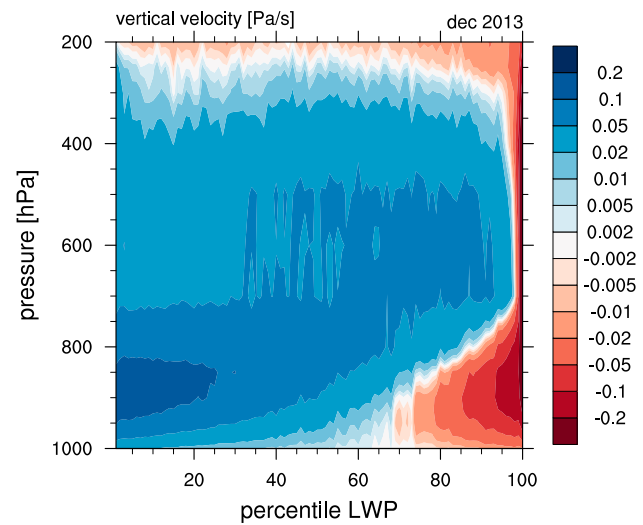

(c) 10
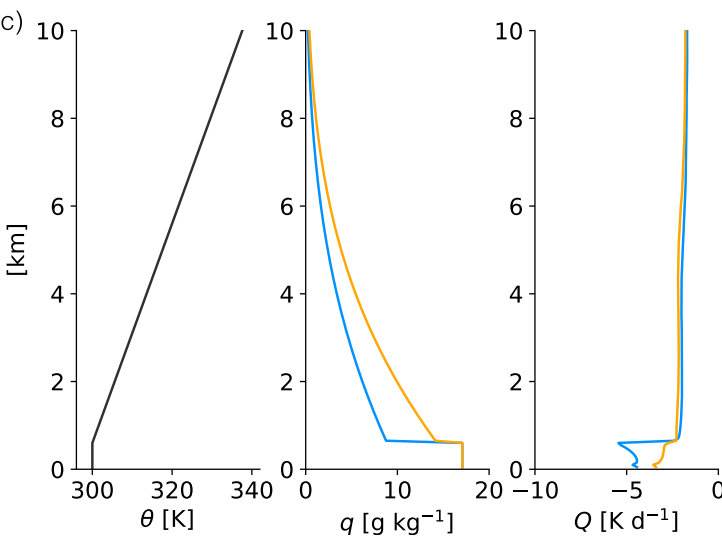

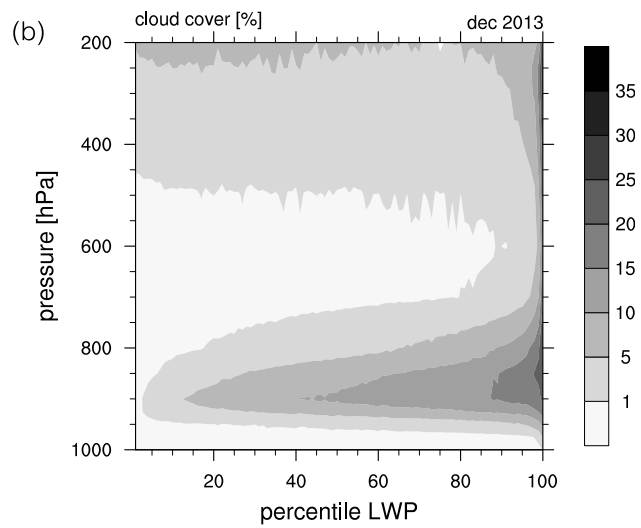

$(d)$

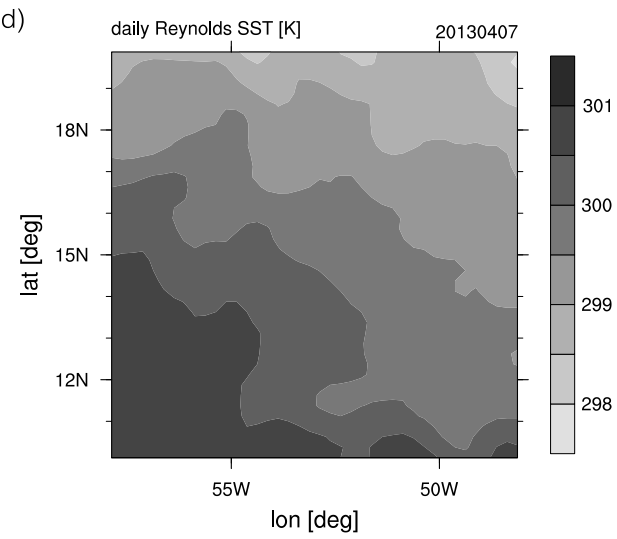

FIG. 1. (a) Pressure velocity and (b) cloud fraction in $1^{\circ} \times 1^{\circ}$ subdomains sorted by LWP percentiles. The analyses is based on simulations performed for December 2013 with 1.25-km grid spacing (Klocke et al. 2017) and is limited to a subtropical region dominated by shallow clouds $\left(12^{\circ}-17^{\circ} \mathrm{N}, 63^{\circ}-43^{\circ} \mathrm{W}\right)$. Typical (c) radiation profile and (d) SST distribution for a subtropical region dominated by large-scale subsidence and shallow convection. (c) Potential temperature and humidity profiles used to calculate longwave radiative cooling rates. (d) SST distribution for 7 Apr 2013 from the Reynolds SST dataset (Reynolds et al. 2007) with an SST difference of $2.9 \mathrm{~K}$ across the region. Monthly mean Reynolds SSTs for a 10-yr period (2007-16) show that the largest SST differences in a $10^{\circ} \times 10^{\circ}$ area $\left(10^{\circ}-20^{\circ} \mathrm{N}, 58^{\circ}-48^{\circ} \mathrm{W}\right)$ appear in April. The daily Reynolds SST for all Aprils in the same period and area gives a mean SST difference of $2.9 \pm 0.5 \mathrm{~K}$ for a characteristic length scale of $1000 \mathrm{~km}$. This translates to a typical SST gradient of $0.6 \mathrm{~K}(200 \mathrm{~km})^{-1}$.

nonconvective regions around $800 \mathrm{hPa}$, and an inflow at the surface (Stevens et al. 2001; Bretherton and Blossey 2017).

The causes of these shallow mesoscale circulations are still a matter of some debate. Idealized studies show that to cause a shallow circulation instead of a deep circulation a heating needs to be placed within the BL instead of above it (Nicholls et al. 1991; Wu et al. 2000; Wu 2003; Bellon et al. 2017; Stevens et al. 2017). In an influential paper, Lindzen and Nigam (1987) proposed that surface winds are driven by sea surface temperature (SST) differences through baroclinic pressure gradients that develop as the SST imprints its temperature on the BL. This sea-breeze-like mechanism has been confirmed by many studies (e.g., Emanuel et al. 1994; Sobel and Neelin 2006; Nolan et al. 2007). However, typical SST gradients for subsidence-dominated shallow cumulus regimes are rather low, around $0.3 \mathrm{~K}$ $(100 \mathrm{~km})^{-1}$ (Fig. 1d). Besides SST differences, spatial differences in low-level radiative cooling are expected to drive shallow circulations (Nigam 1997; Wang et al. 2005; Nishant et al. 2016; Naumann et al. 2017). In subsidence regions with a dry free troposphere and a moist $\mathrm{BL}$, low-level radiative cooling is strong because the moist and warm BL strongly emits longwave radiation but relatively little returns to the BL top from the dry layer above (Fig. 1c). For a weaker humidity inversion due to moister air above the $\mathrm{BL}$, the radiative BL cooling is considerably weaker. Also, the presence of shallow cumulus clouds modifies the radiative profile and tends to weaken radiative cooling at cloud base. A typical value of a difference of radiative cooling 
averaged over the BL depth between dry and moist regions is around $3 \mathrm{~K} \mathrm{day}^{-1}$ but might vary, mostly as a function of the moisture content above the inversion (Mapes and Zuidema 1996; Stevens et al. 2017). Differential low-level radiative cooling affects the BL temperature structure, which in turn causes a pressure-driven flow. Therefore differential low-level radiative cooling is a potential candidate to drive a shallow circulation.

In addition to the subsidence regime dominated by shallow convection, shallow circulations have also been found in connection with deep convection such as in the vicinity of the intertropical convergence zone (ITCZ; e.g., Trenberth et al. 2000; Zhang et al. 2008; Back and Bretherton 2009; Fläschner et al. 2018; Schulz and Stevens 2018) and in idealized simulation of radiativeconvective equilibrium (RCE; e.g., Bretherton et al. 2005; Muller and Held 2012; Wing and Emanuel 2013). Although there might be driving mechanisms common to shallow circulations in subsidence regimes and shallow circulations in regimes of deep convection, the latter are not the motivation of this study and results may not be directly transferable as we expect the cloud layer to play a more active role in connection with deep convection.

The formulation of bulk conceptual models to describe the tropical atmosphere has a long tradition in atmospheric science, both for describing the interaction of deep and shallow convection in the tropics via a deep circulation but also focusing on a particular regime such as shallow convection in a homogeneous setup (e.g., Lilly 1968; Tennekes 1973; Sarachik 1978; Albrecht et al. 1979; Betts 1985; Betts and Ridgway 1989; Nilsson and Emanuel 1999; Betts 2000; Stevens 2006). Apart from the recent study by Naumann et al. (2017), which only explores the dry case, these bulk conceptual models have not been applied to investigate the behavior of radiatively driven shallow circulations.

In this paper, we focus on the structure of the shallow cumulus-topped BL and on shallow circulations that are situated entirely in a region of steady subsidence and are driven either by spatial differences in radiative BL cooling or by spatial differences in SST. The goal of the study is to develop a conceptual understanding of the interaction between moisture and convection, surface forcing, BL properties, and shallow circulations in a trade wind-like regime. A prerequisite for a conceptual model that can represent heterogeneities (in radiative cooling or SST) and hence a shallow circulation is a conceptual model that can represent the response of the atmosphere to a homogeneous forcing. It turns out that the homogeneous setup of the model we introduce is able to explain interesting aspects of the shallow cumulus-topped BL, such as the omnipresence of a cumulus mass flux over the ocean or the little variation in cloud-base height, and thereby confirms and complements pioneering studies from Albrecht et al. (1979) and Betts and Ridgway (1989).

It is therefore worthwhile to analyze the homogeneous setup for its own sake, and as a reference for the heterogeneous model to be able to isolate the effect of the shallow circulation on the $\mathrm{BL}$ and on convection. Doing so, we ask the following questions: How does the effect of moisture and convection modify the BL structure? How effective are gradients in radiative cooling in driving convection and shallow circulations? Is the strength of a shallow circulation driven by differences in radiative $\mathrm{BL}$ cooling comparable to the strength of a shallow circulation driven by differences in SST?

To answer these questions, we extend a conceptual model for a dry BL (Naumann et al. 2017) to include, in the simplest possible way, effects of moisture and shallow convection. The bulk conceptual model applies the weak temperature gradient assumption in the free troposphere and formulates budget equations for the BL height, the BL temperature, and the BL humidity and a momentum equation for the BL flow. To represent the effect of shallow convection, a simple formulation for the convective mass flux is used, which relaxes the BL height to the lifting condensation level and ventilates the BL. In the bulk conceptual model, boundary conditions prescribe differences in radiative BL cooling or differences in SST according to those typically found in subsidencedominated regions with shallow clouds (Figs. 1c,d).

This manuscript has two parts: First in section 2, we formulate a homogeneous (one-column) model to assess the BL properties and the effect of moisture and convection in the absence of a shallow circulation. The second part, section 3, addresses a two-column setup, where differential radiative cooling or SST differences cause a pressure-driven shallow circulation, which couples the cold and the warm column dynamically.

\section{Homogeneous setup}

The conceptual model developed in this section is an extension of a homogeneous, one-column, dry conceptual model (Naumann et al. 2017) but includes the effects of moisture and a simple formulation of a convective mass flux that ventilates $\mathrm{BL}$ air to the free troposphere (FT) above. The model is representative for a region dominated by shallow convection and large-scale subsidence. In the planetary BL conserved variables are well mixed. Subsidence dries the air above the BL and vigorous mixing in the $\mathrm{BL}$ sharpens the $\mathrm{BL}$ inversion from below, which allows for strong radiative cooling at the BL top (Fig. 1c). In this section we analyze how increasing radiative $\mathrm{BL}$ cooling affects the homogeneous 
BL. In particular we show that the inclusion of moisture yields a distinct $\mathrm{BL}$ response as the convective mass flux strongly regulates the BL properties and balance.

\section{a. Formulation of a moist conceptual model}

The homogeneous conceptual model consists of two layers, the BL and a layer above the inversion (Fig. 2). We denote the latter with the subscript FT to have a consistent naming both for the dry case and the moist case but in the moist model the layer above the inversion may also be characterized as a cloud layer if the convective mass flux is found to be nonzero. In that case, the BL corresponds to the subcloud layer. In the $\mathrm{BL}$, the potential temperature $\theta_{\mathrm{BL}}$ and water vapor mixing ratio $q_{\mathrm{BL}}$ are well mixed. We prescribe distinct radiative cooling rates both in the $\mathrm{BL}$ and the $\mathrm{FT}, Q_{\mathrm{BL}}$ and $Q_{\mathrm{FT}}$, respectively, as well as the temperature profile in the FT $\theta_{\mathrm{FT}}$, the surface temperature $\theta_{\mathrm{sfc}}$, and the humidity jump at the boundary between the BL and the FT $\Delta q$. The surface is assumed to be saturated with surface water vapor mixing ratio, $q_{\mathrm{sfc}}=q_{\mathrm{sat}}\left(\theta_{\mathrm{sfc}}, z=0\right)$. This yields three prognostic equations for $\theta_{\mathrm{BL}}, q_{\mathrm{BL}}$, and the BL height, $h$ [Eqs. (1), (4), and (7), respectively]:

$$
\frac{\partial \theta_{\mathrm{BL}}}{\partial t}=Q_{\mathrm{BL}}+\frac{1}{h}\left(w_{e} \Delta \theta+F_{\theta}\right),
$$

with

$$
\begin{aligned}
& \Delta \theta=\theta_{0}+\Gamma h-\theta_{\mathrm{BL}}, \\
& F_{\theta}=C_{d} V\left(\theta_{\mathrm{sfc}}-\theta_{\mathrm{BL}}\right) ;
\end{aligned}
$$

$$
\frac{\partial q_{\mathrm{BL}}}{\partial t}=\frac{1}{h}\left(w_{e} \Delta q+F_{q}\right),
$$

with

$$
\begin{aligned}
\Delta q & =q_{\mathrm{FT}}-q_{\mathrm{BL}}, \\
F_{q} & =C_{d} V\left(q_{\mathrm{sfc}}-q_{\mathrm{BL}}\right) ; \quad \text { and }
\end{aligned}
$$

$$
\frac{\partial h}{\partial t}=w_{\mathrm{FT}}+w_{e}+w_{m}=\frac{Q_{\mathrm{FT}}}{\Gamma}+A \frac{F_{B}}{\Delta \theta_{v}}+w_{m},
$$

with

$$
\begin{aligned}
\Delta \theta_{v} & =\left(\theta_{0}+\Gamma h\right)\left(1+\varepsilon q_{\mathrm{FT}}\right)-\theta_{v, \mathrm{BL}}, \\
F_{B} & =F_{\theta}+\varepsilon \theta_{\mathrm{BL}} F_{q}, \\
w_{m} & =\left\{\begin{array}{ccc}
-\frac{h-\mathrm{LCL}}{\tau} & \text { if } & \mathrm{LCL}<h \\
0 & \text { if } & \mathrm{LCL} \geq h
\end{array} .\right.
\end{aligned}
$$

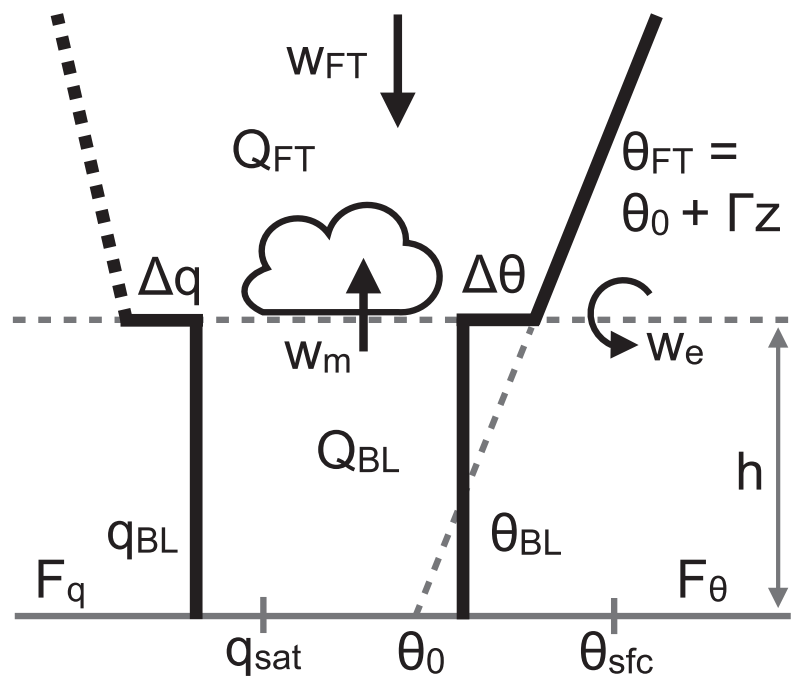

FIG. 2. Sketch of the moist one-column model.

Here, $\Delta \theta$ is the temperature inversion strength, $\theta_{0}$ a reference surface temperature to determine $\theta_{\mathrm{FT}}(z \geq h)=$ $\theta_{0}+\Gamma z$ with $\Gamma=\left.(\partial \theta / \partial z)\right|_{\mathrm{FT}}$ the potential temperature gradient in the FT; $q_{\mathrm{FT}}$ is the free-tropospheric water vapor mixing ratio just above the $\mathrm{BL}$ inversion, $F_{\theta}$ the kinematic surface sensible heat flux, $F_{q}$ the kinematic surface moisture flux, $F_{B}$ the kinematic buoyancy flux, $C_{d}$ the drag coefficient, and $V$ the background wind speed, which can be associated with a large-scale circulation, for example, caused by remote deep convective regions or meridional pressure gradients. Prescribed parameters are chosen to represent typical values in the subsidence-dominated shallow cumulus regime of the trades (as shown in Fig. 1). They are equal to those used in Naumann et al. (2017) and are given in Table 1.

Equation (1) describes the BL budget of $\theta_{\mathrm{BL}}$, which is modified by radiative cooling, entrainment at the BL top, and the surface sensible heat flux. Equation (2) gives a geometrical constraint on $\Delta \theta$ and Eq. (3) is a common bulk-aerodynamic closure for $F_{\theta}$ (e.g., Stevens 2006). Equation (4) describes the BL budget of $q_{\mathrm{BL}}$, which depends on a balance between entrainment drying and surface moistening. Equation (5) defines $\Delta q$ and Eq. (6) gives a common closure for $F_{q}$.

Equation (7) expresses that $h$ is determined by a balance of the large-scale subsidence velocity $w_{\mathrm{FT}}$ $\left(<0 \mathrm{~cm} \mathrm{~s}^{-1}\right)$, the entrainment velocity at the inversion $w_{e}\left(>0 \mathrm{~cm} \mathrm{~s}^{-1}\right)$, and the convective mass flux velocity $w_{m}\left(<0 \mathrm{~cm} \mathrm{~s}^{-1}\right)$. The entrainment velocity, $w_{e}=A F_{B} / \Delta \theta_{v}$, is given by a commonly used closure assumption with the inversion entrainment efficiency, $A=0.41$, being 
TABLE 1. Prescribed parameters used in this study if not explicitly stated otherwise.

\begin{tabular}{lc}
\hline \hline Parameter & Value \\
\hline$Q_{\mathrm{BL}}$ & -1.0 to $-6.0 \mathrm{~K} \mathrm{day}^{-1}$ \\
$Q_{\mathrm{FT}}$ & $-1.0 \mathrm{~K} \mathrm{day}^{-1}$ \\
$\Gamma$ & $5.0 \mathrm{~K} \mathrm{~km}^{-1}$ \\
$\theta_{0}$ & $298.0 \mathrm{~K}$ \\
$\theta_{\mathrm{sfc}}$ & $301.0 \mathrm{~K}$ \\
$A$ & 0.41 \\
$C_{d}$ & 0.001 \\
$V$ & $5 \mathrm{~m} \mathrm{~s}^{-1}$ \\
$\tau$ & $\left.15 \mathrm{~min}^{-1}\right)$ \\
$\Delta q$ & $-\min \left(q_{\mathrm{BL}}, 3 \mathrm{~g} \mathrm{~kg}^{-1}\right)$ \\
Two-column model only & \\
$X_{p}$ & $20 \mathrm{~km}$ \\
$X_{1}=X_{2}$ & $100 \mathrm{~km}$ \\
\hline
\end{tabular}

set by the flow (Deardorff et al. 1974; Naumann et al. 2017). The large-scale subsidence velocity, $w_{\mathrm{FT}}=Q_{\mathrm{FT}} / \Gamma$, is determined by the weak temperature gradient assumption (Sobel and Bretherton 2000), which is valid in the FT but not in the BL. In the cloud layer, turbulent convective transport also plays a role in the heat budget but is neglected here, which leads to an overestimation of $w_{\mathrm{FT}}$ in the cloud layer. We show in section $2 \mathrm{~d}$ that although $w_{\mathrm{FT}}$ is overestimated in the cloud layer, it only plays a minor role in Eq. (7). Betts and Ridgway (1989) specified a stronger $\left(-0.5 \mathrm{~cm} \mathrm{~s}^{-1}\right)$ large-scale subsidence velocity. The values we choose $\left(w_{\mathrm{FT}}=Q_{\mathrm{FT}} / \Gamma=-0.23 \mathrm{~cm} \mathrm{~s}^{-1}\right.$; see Table 1$)$ are in the range of what is simulated using general circulation models (Fläschner et al. 2018), albeit on the low end. Choosing a value on the low end of the range helps maintain consistency with the study using the dry model (Naumann et al. 2017) and allows a better differentiation between moist and dry columns.

Equations (8) and (9) follow from the definition of the virtual potential temperature, $\theta_{v}=\theta(1+\varepsilon q)$, where $\varepsilon=0.61$. Note that $\rho c_{p} F_{B}=\rho c_{p} F_{\theta}+\left(\varepsilon \theta_{\mathrm{BL}} c_{p} / L\right) \rho L F_{q}=$ $\mathrm{SH}+0.07 \mathrm{LH}$, where $\mathrm{SH}$ is the dynamic sensible heat flux $\left(\mathrm{W} \mathrm{m}^{-2}\right)$ and $\mathrm{LH}$ is the dynamic latent heat flux $\left(\mathrm{W} \mathrm{m}^{-2}\right)$.

A simple formulation of $w_{m}$ is given in Eq. (10). The convective mass flux velocity relaxes $h$ to the lifting condensation level (LCL) with a time scale of $\tau=15 \mathrm{~min}$ (Neggers et al. 2006), if the LCL is smaller than $h$. Apart from the relaxation time scale, which is a prescribed parameter in our model, this formulation corresponds to the approach of Betts (2000), which directly enforces $h$ to match LCL (Albrecht et al. 1979; Stevens et al. 2001; Lareau et al. 2018). The LCL is calculated for each temperature and humidity profile, where the pressure at the LCL is obtained by integrating downward assuming

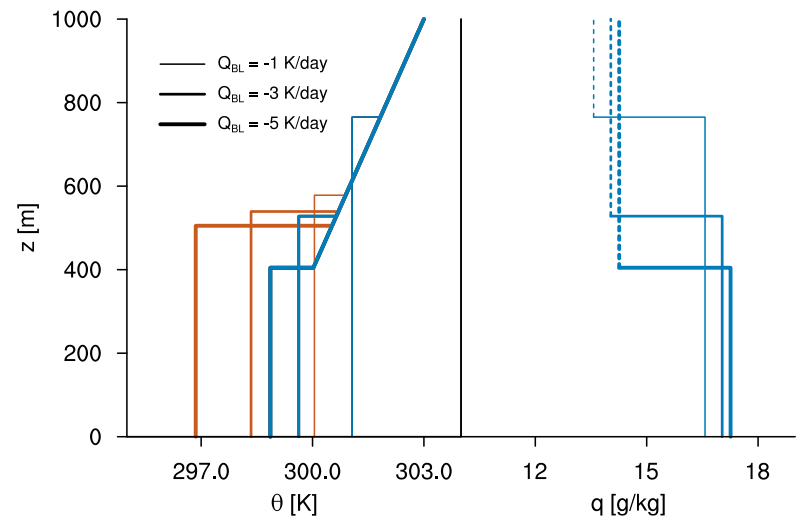

FIG. 3. Equilibrium profiles of the dry (brown) and the moist (blue) one-column model. Above the inversion, $q$ is not declared in the conceptual model (dashed).

a constant and prescribed pressure of $850 \mathrm{hPa}$ at $1500 \mathrm{~m}$. We assume that convection ventilates the $\mathrm{BL}$ in the sense that it transports BL air (with mean BL air properties) above the inversion. Therefore $w_{m}$ does not affect the BL heat and moisture budget directly [Eqs. (1) and (4)]. Other indirect effects such as evaporative cooling and moistening of BL air through precipitation are also neglected.

Compared to the dry conceptual model and in addition to $\tau$, the moist model contains a second additional, prescribed parameter: $\Delta q$. We choose $\Delta q=$ $q_{\mathrm{FT}}-q_{\mathrm{BL}}=-\min \left(q_{\mathrm{BL}}, 3 \mathrm{~g} \mathrm{~kg}^{-1}\right)$; that is, we prescribe a fixed humidity inversion strength of $\Delta q=-3 \mathrm{~g} \mathrm{~kg}^{-1}$ for $q_{\mathrm{BL}} \geq 3 \mathrm{~g} \mathrm{~kg}^{-1}$ similar to Betts (2000). The formulation of $w_{m}$ and $\Delta q$ can be interpreted as a crude cloud model whereby clouds maintain $\Delta q$. To a first approximation $q_{\mathrm{FT}}$ is related to $q_{\mathrm{BL}}$ because convection transports $\mathrm{BL}$ air from the BL to above the inversion. With this simple formulation we aim to include the effects of moisture in a way that contains as few additional degrees of freedom as possible, and deliberately avoids an explicit representation of the cloud layer so as to build intuition as to what the important balances are.

In the conceptual model, a radiative $\mathrm{BL}$ cooling rate $Q_{\mathrm{BL}}$ is prescribed [Eq. (1)]. This choice implies that the radiative flux divergence integrated over the BL $F_{\text {rad }}$ decreases for a shallowing BL. Another choice would be to assume a constant $F_{\text {rad }}$, which is independent of $h$ but translates into an increase in $Q_{\mathrm{BL}}$ with decreasing $h$. Prescribing $F_{\text {rad }}$ instead of $Q_{\mathrm{BL}}$, however, does not change the overall results of the model except for a linear relation found for the change of $h$ as a function of the prescribed $F_{\text {rad }}$. Prescribing $Q_{\mathrm{BL}}$ instead of $F_{\mathrm{rad}}$ results in a nonlinear relation for the change of $h$ as a function of the prescribed $Q_{\mathrm{BL}}$ (see Fig. 3). In the following we describe results only for a prescribed $Q_{\mathrm{BL}}$. 

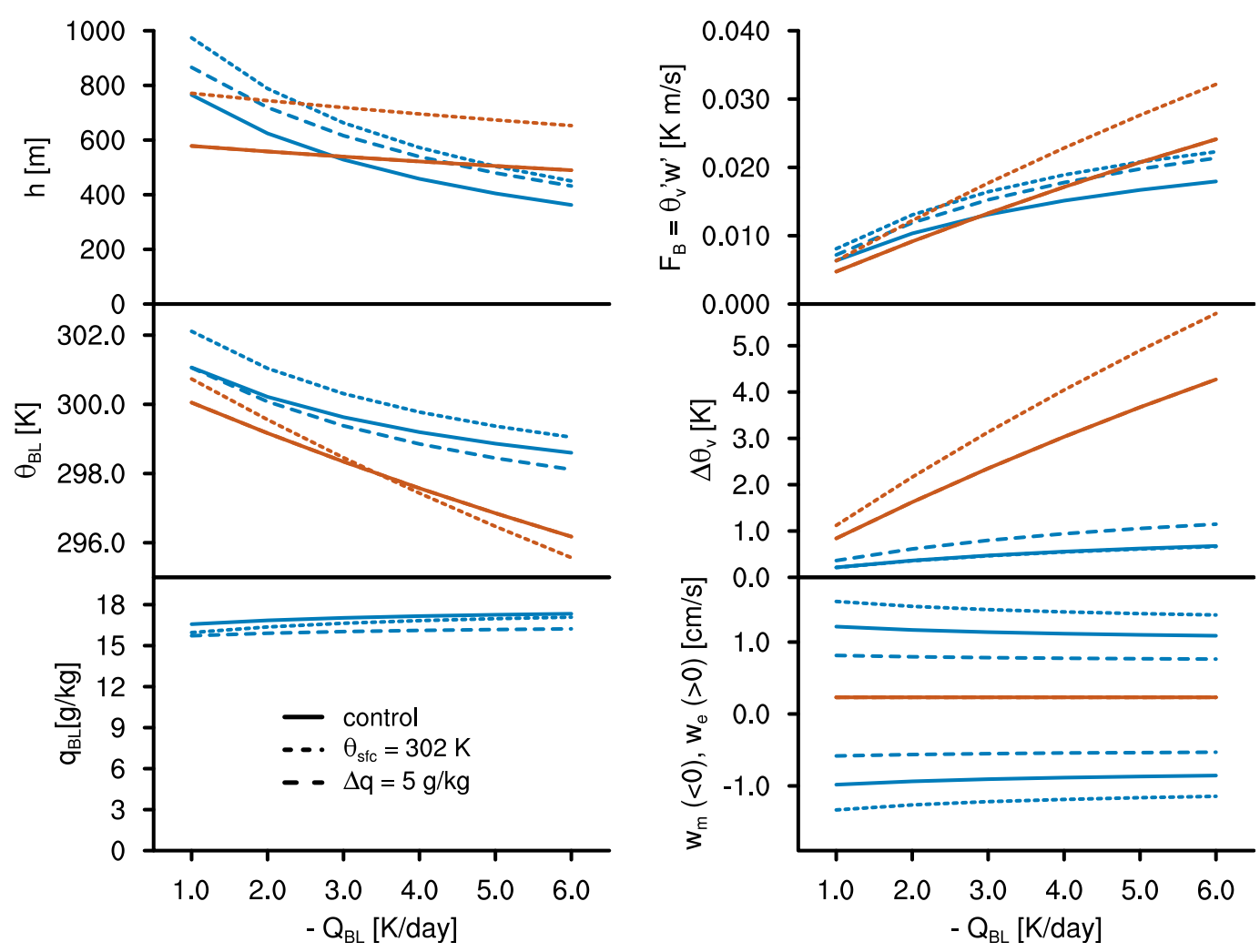

FIG. 4. Equilibrium solution of the dry and the moist one-column model. Moist case in blue, dry case in brown. For the control case $\theta_{\mathrm{sfc}}=301.0 \mathrm{~K}$ and $\Delta q=3 \mathrm{~g} \mathrm{~kg}^{-1}$.

As initial conditions for the homogeneous moist conceptual model, we use the equilibrium solution of the dry BL by solving Eq. (1) to Eq. (10) with $q_{\mathrm{BL}}=$ $q_{\mathrm{FT}}=0 \mathrm{~g} \mathrm{~kg}^{-1}$ and $F_{q}=0 \mathrm{~g} \mathrm{~kg}^{-1} \mathrm{~m} \mathrm{~s}^{-1}$ (hence also $w_{m}=$ $0 \mathrm{~cm} \mathrm{~s}^{-1}$ ); that is, the prognostic equations for $\theta_{\mathrm{BL}}$ and $h$, Eqs. (1) and (7), respectively, are set equal to zero. This equilibrium solution of the dry BL is equivalent to the solution of the homogeneous dry conceptual model as formulated in Naumann et al. (2017). The stationary, equilibrium solution of the moist conceptual model is then obtained by integrating the prognostic equations [Eqs. (1)-(10)], starting from these initial conditions until a new equilibrium is reached. We use an integration time step of $5 \mathrm{~min}$. The equilibrium solution of the moist conceptual model is independent of the initial conditions for a wide range of physically sensible initial conditions. In this study, we focus on the stationary solution. The transient response from the initially dry BL to the moist $\mathrm{BL}$ is included in appendix A. While the dry model's equilibrium can be calculated analytically, this is not directly possible for the moist conceptual model because the LCL needs to be calculated iteratively. Most of this study applies the iterative solution, with the exception of section $2 \mathrm{c}$, where we linearize the model's equations around a given state to analyze the change in state due to a radiative perturbation.

\section{b. Characteristics of the observed trade wind regime}

Integrating the three prognostic equations [Eqs. (1), (4), and (10)] to stationarity allows us to analyze the equilibrium state of the moist conceptual model and to compare it to observations from the trades: In the conceptual model, the LCL is located roughly between 400 and $1000 \mathrm{~m}$ for different large-scale conditions (Fig. 3; see also Fig. 4). This agrees well with the mean cloud-base height of active shallow clouds at the Barbados Cloud Observatory (Stevens et al. 2016), which is rather invariable around a mean of $740 \pm 340 \mathrm{~m}( \pm 2 \sigma$, O. Tessiot 2018, personal communication), and with earlier conceptual models, which apply a more complex cloud layer (Albrecht et al. 1979; Betts and Ridgway 1989). The near-surface relative humidity in the conceptual model is between $72 \%$ and $85 \%$ for the cases shown in Fig. 3, while it is uniformly found between $70 \%$ and $90 \%$ in observations at Barbados (Lonitz et al. 2015; Klingebiel et al. 2019). Assuming no lateral inflow or outflow, the equilibrium surface precipitation in the moist conceptual model can be estimated from the surface moisture flux and is found to be $3.5-3.9 \mathrm{~mm} \mathrm{day}^{-1}$ 
for the cases shown in Fig. 3. A typical area-averaged surface precipitation rate in a field of shallow cumulus in the trades is around $1-3 \mathrm{~mm}^{-1 a y}{ }^{-1}$ (Rauber et al. 2007; Seifert et al. 2015). We therefore conclude that the conceptual model is able to reproduce key characteristics of the observed trade wind shallow cumulus regime.

\section{c. Linear solution for a radiative perturbation around a given state}

A great deal of insight as to the behavior of the model and, by inference, the balances in the trades can be extracted by linearizing about the equilibrium solution to Eqs. (1)-(7). From the entropy $\theta$ and moisture balance we have

$$
\begin{aligned}
-h Q_{\mathrm{BL}} & =w_{e}\left(\theta_{0}+\Gamma h-\theta_{\mathrm{BL}}\right)+C_{d} V\left(\theta_{\mathrm{sfc}}-\theta_{\mathrm{BL}}\right), \\
q_{\mathrm{BL}} & =\frac{w_{e}}{C_{d} V} \Delta q+q_{\mathrm{sfc}} .
\end{aligned}
$$

In addition, by assuming that

$$
q_{\mathrm{BL}} \approx q_{\mathrm{sat}}\left(\theta_{\mathrm{BL}}, h\right),
$$

which implies that $h \approx \mathrm{LCL}$, the parameterized form of $w_{e}$, that is, an expression of $h$, is

$$
\begin{aligned}
& w_{e}\left(\theta_{0}+\Gamma h-\theta_{\mathrm{BL}}+\varepsilon \theta_{\mathrm{BL}} \Delta q\right) \\
& \quad=A C_{d} V\left[\left(\theta_{\mathrm{sfc}}-\theta_{\mathrm{BL}}\right)+\varepsilon \theta_{\mathrm{BL}}\left(q_{\mathrm{sfc}}-q_{\mathrm{BL}}\right)\right],
\end{aligned}
$$

where we use $\left(\theta_{0}+\Gamma h\right) q_{\mathrm{FT}}-\theta_{\mathrm{BL}} q_{\mathrm{BL}} \approx \theta_{\mathrm{BL}} \Delta q$ because $\Delta \theta / \theta_{\mathrm{BL}} \ll \Delta q / q_{\mathrm{BL}}$.

Equations (11)-(14) are linearized around this equilibrium for a change due to a radiative perturbation $\delta Q_{\mathrm{BL}}$ :

$$
\begin{aligned}
- & \delta h\left(Q_{\mathrm{BL}}+\Gamma w_{e}\right)-\delta Q_{\mathrm{BL}} h \\
& =\delta w_{e}\left(\theta_{0}+\Gamma h-\theta_{\mathrm{BL}}\right)-\delta \theta_{\mathrm{BL}}\left(C_{d} V+w_{e}\right), \\
\delta q_{\mathrm{BL}} & =\delta w_{e}\left(\frac{\Delta q}{C_{d} V}\right), \\
\delta q_{\mathrm{BL}} & =\mathscr{C} q_{\mathrm{BL}}\left(\delta \theta_{\mathrm{BL}}+\gamma_{\mathrm{dry}} \delta h\right),
\end{aligned}
$$

$$
\begin{aligned}
\delta w_{e}\left(\theta_{0}+\Gamma h-\theta_{\mathrm{BL}}+\varepsilon \theta_{\mathrm{BL}} \Delta q\right)= & \delta \theta_{\mathrm{BL}}\left\{w_{e}(1-\varepsilon \Delta q)-A C_{d} V\left[1-\varepsilon\left(q_{\mathrm{sfc}}-q_{\mathrm{BL}}\right)\right]\right\} \\
& -\delta h\left(w_{e} \Gamma\right)-\delta q_{\mathrm{BL}}\left(A C_{d} V \varepsilon \theta_{\mathrm{BL}}\right),
\end{aligned}
$$

with $\mathscr{C}=\left(1 / q_{\mathrm{sat}}\right)\left(\partial q_{\mathrm{sat}} / \partial T\right)=l_{v} /\left(R_{v} T^{2}\right)$ given by the Clausius-Clapeyron equation and $\gamma_{\text {dry }}=\left.(\partial T / \partial z)\right|_{\text {dry }}$ as the dry adiabatic temperature gradient. Solving Eq. (15) to Eq. (18) for $\delta h, \delta \theta_{\mathrm{BL}}, \delta q_{\mathrm{BL}}$, and $\delta w_{e}$, simplifying the result by neglecting comparatively small terms, and nondimensionalizing the results yields

$$
\begin{aligned}
\frac{\delta \theta_{\mathrm{BL}}}{\Gamma h} & =\frac{1}{Q_{\mathrm{turb}}-Q_{\mathrm{BL}}} \delta Q_{\mathrm{BL}}, \\
\frac{\delta h}{h} & =\frac{1}{Q_{\mathrm{turb}}-Q_{\mathrm{BL}}} \delta Q_{\mathrm{BL}}=\frac{\delta \theta_{\mathrm{BL}}}{\Gamma h}, \\
\frac{\delta q_{\mathrm{BL}}}{q_{\mathrm{BL}}} & =\mathscr{C} \frac{\left(\Gamma+\gamma_{\mathrm{dry}}\right) h}{Q_{\mathrm{turb}}-Q_{\mathrm{BL}}} \delta Q_{\mathrm{BL}}=\mathscr{C} \frac{\Gamma+\gamma_{\mathrm{dry}}}{\Gamma} \delta \theta_{\mathrm{BL}}, \\
\frac{\delta w_{e}}{w_{e}} & =\mathscr{C} \frac{C_{d} V q_{\mathrm{BL}}\left(\Gamma+\gamma_{\mathrm{dry}}\right) h}{w_{e} \Delta q\left(Q_{\mathrm{turb}}-Q_{\mathrm{BL}}\right)} \delta Q_{\mathrm{BL}}=\frac{C_{d} V}{w_{e} \Delta q} \delta q_{\mathrm{BL}},
\end{aligned}
$$

where $Q_{\text {turb }}=C_{d} V \Gamma(A+1)$ can be interpreted as a turbulent $\mathrm{BL}$ heating term related to the turbulent $\mathrm{BL}$ flux divergence due to surface fluxes and inversion entrainment. Inserting values from the control case with
$Q_{\mathrm{BL}}=-3 \mathrm{~K} \mathrm{day}^{-1}$ (Table 1) and a radiative perturbation of $\delta Q_{\mathrm{BL}}=-1 \mathrm{~K} \mathrm{day}^{-1}$ gives a decrease in the normalized BL temperature of $20 \%$; that is, $\delta \theta_{\mathrm{BL}} /(\Gamma h) \approx-0.2$. Accordingly we find $\delta h / h \approx-0.2, \delta q_{\mathrm{BL}} / q_{\mathrm{BL}} \approx 0.03$, and $\delta w_{e} / w_{e} \approx-0.1$. Hence, for a perturbation in the radiative BL cooling, it is mostly $\theta_{\mathrm{BL}}$ and $h$ that adapt while changes in $q_{\mathrm{BL}}$ and $w_{e}$ are rather small. Despite this and as further explained below, the presence of $q_{\mathrm{BL}}$ affects the sensitivity of $h$ on $Q_{\mathrm{BL}}$. These results of the linearized model are in very good qualitative and quantitative agreement with the iterative solution of the full model system (Figs. 3, 4) and also agree with the mixedlayer model of Betts (2000) for a constant vegetative resistance.

We can interpret the linearized model as follows: Perturbing the system by increasing the radiative BL cooling leads to a decrease in $\theta_{\mathrm{BL}}$ [Eq. (19)]. This decrease in $\theta_{\mathrm{BL}}$ depends on the sum of turbulent $\mathrm{BL}$ heating and radiative $\mathrm{BL}$ cooling and is stronger for stronger stability $(\Gamma h)$. When $\theta_{\mathrm{BL}}$ decreases due to stronger radiative $\mathrm{BL}$ cooling, the LCL decreases and $w_{m}$ adjusts $h$ to the LCL. In the linear limit, $h$ decreases 
by $\Gamma^{-1} \delta \theta_{\mathrm{BL}}$ [Eq. (20)], so that $\Delta \theta$ is kept constant. A change in $\theta_{\mathrm{BL}}$ leads to a change in $q_{\mathrm{sat}}$ at the BL top of $\mathscr{C}\left[\left(\Gamma+\gamma_{\mathrm{dry}}\right) / \Gamma\right] \delta \theta_{\mathrm{BL}}$ [Eq. (21)], which determines the increase in $q_{\mathrm{BL}}$. Hence, the change in $q_{\mathrm{BL}}$ ensures that the LCL matches $h$ in the new state as demanded in Eq. (13). The factor $\left(\Gamma+\gamma_{\text {dry }}\right) / \Gamma$ reflects the offsetting effect of the change in $h$ on $\theta_{\mathrm{BL}}$; that is, reducing $\theta_{\mathrm{BL}}$ cools the BL top (where the LCL condition is valid) but reducing $h$ increases the absolute temperature at the BL top. These two effects work against each other and imply less of a reduction in $q_{\mathrm{BL}}$. In that sense, $w_{m}$ acts as a moisture valve to the $\mathrm{BL}$ varying $h$ while keeping $q_{\mathrm{BL}}$ roughly constant. The small increase in $q_{\mathrm{BL}}$ requires less ventilation of dry, free-tropospheric air into the $\mathrm{BL}$, that is, a small decrease in $w_{e}$ of $C_{d} V /\left[(\Delta q) \delta q_{\mathrm{BL}}\right]$ [Eq. (22)]. Because $w_{e}$ is roughly balanced by $w_{m}$ (see section $2 \mathrm{~d}), w_{m}$ also only weakly changes with the strength of the radiative BL cooling.

For the dry BL, the analogous procedure gives

$$
\begin{aligned}
\frac{\delta \theta_{\mathrm{BL}}}{\Gamma h} & =\frac{1}{Q_{\mathrm{turb}}-Q_{\mathrm{BL}}\left(1+A C_{d} V \Gamma / Q_{\mathrm{FT}}\right)} \delta Q_{\mathrm{BL}}, \\
\frac{\delta h}{h} & =\frac{1}{\left(1+A C_{d} V \Gamma / Q_{\mathrm{FT}}\right)^{-1} Q_{\mathrm{turb}}-Q_{\mathrm{BL}}} \delta Q_{\mathrm{BL}} .
\end{aligned}
$$

The linearized solution of the dry case differs from the linearized solution of the moist case only by the term $\left(1+A C_{d} V \Gamma / Q_{\mathrm{FT}}\right)$, which determines whether $h$ decrease or increases with stronger radiative $\mathrm{BL}$ cooling and is related to the strength of the surface coupling [see section 2b(1) in Naumann et al. 2017]. In the dry BL the constraint that $q_{\mathrm{BL}}$ is equal to $q_{\mathrm{sat}}$ at the LCL does not exist, so that the BL height can rise or sink to allow for the entrainment to balance the FT subsidence velocity, which appears in the solution as $w_{\mathrm{FT}}=Q_{\mathrm{FT}} / \Gamma$. In the linearized solution of the moist BL, $Q_{\mathrm{FT}}$ does not appear and we will bring out in the next section how $w_{\text {FT }}$ plays only a minor role for the moist BL balance.

\section{d. Convective mass flux and its role for the velocity balance}

The main effect of moisture on the BL structure is in the ventilation of the moist $\mathrm{BL}$ through the convective mass flux, which must be balanced by inversion entrainment: Quite different from the case of the moist $\mathrm{BL}$, the equilibrium balance of vertical velocities at the BL top in the dry case is given by a direct balance of $w_{\mathrm{FT}}$ and $w_{e}$ [Eq. (7); Fig. 4]. In this case, both velocities are determined by the radiative cooling and the temperature gradient in the FT $\left(w_{\mathrm{FT}}=-w_{e}=Q_{\mathrm{FT}} / \Gamma\right)$ and are therefore independent of the applied radiative $\mathrm{BL}$ cooling. In the case of the moist $\mathrm{BL}, w_{\mathrm{FT}}$ is still determined by $Q_{\mathrm{FT}} / \Gamma$ but $w_{m}\left(\approx-1 \mathrm{~cm} \mathrm{~s}^{-1}\right)$ is about 4 times larger in magnitude than $w_{\mathrm{FT}}\left(=-0.23 \mathrm{~cm} \mathrm{~s}^{-1}\right)$. Ventilating BL air into the free troposphere above, $w_{m}$ acts as a moisture valve for the $\mathrm{BL}$ and provides a negative tendency for $\partial h / \partial t$ [Eq. (7)]. To balance $w_{m}$ and thereby stabilize $h, w_{e}$ increases compared to the case of the dry BL. Physically, the increase of $w_{e}$ in the moist $\mathrm{BL}$ as compared to the dry BL can be interpreted by noting that $h$ decreases in the transient response from the dry to the moist BL as soon as the convective mass flux sets in (Fig. A1). As $h$ decreases, $\mathrm{SH}$ is more effective in warming the shallower BL. Both the increase in $\theta_{\mathrm{BL}}$ and the decrease in $h$, decrease the inversion strength ( $\Delta \theta$, also $\left.\Delta \theta_{v}\right)$. If $\Delta \theta_{v}$ is weaker, updrafts in the BL feel less negative buoyancy as they arrive at the inversion and may therefore penetrate deeper and hence increase $w_{e}$.

The minor role of $w_{\mathrm{FT}}$ in the case of the moist $\mathrm{BL}$ explains the model's insensitivity to moderate changes of $Q_{\mathrm{FT}}$ (not shown) and the good agreement with Betts (2000) despite a difference in the prescribed $Q_{\mathrm{FT}}$ between both studies. The results are also in good agreement with large-eddy simulations of shallow cumulus, where the convective mass flux is found to largely balance entrainment deepening of the BL (Stevens et al. 2001). Because the main balance in the case of the moist BL is between $w_{m}$ and $w_{e}$, the insensitivity of $w_{e}$ to the applied radiative $\mathrm{BL}$ cooling translates to an insensitivity of $w_{m}$; that is, stronger radiative cooling does not suppress convection in a homogeneous setup (Fig. 4).

The increase in $w_{e}$ for the moist $\mathrm{BL}$ compared to the dry BL leads to a warming of the moist BL due to stronger entrainment warming $\left(w_{e} \Delta \theta\right.$; Fig. 4$)$. To stabilize $\theta_{\mathrm{BL}}$ despite the high $w_{e}, \Delta \theta$ needs to decrease to lower entrainment warming in the moist BL. A small $\Delta \theta$ is not only needed to support the high $w_{e}$ in the moist equilibrium BL, it also plays a crucial role for the strength of the shallow circulation in section 3 .

Because in equilibrium $\theta_{\mathrm{BL}}$ is higher in the moist model than in the dry model, $\mathrm{SH}$ is smaller in the case of the moist BL than in the dry BL [Eq. (3)]. Additional LH in the moist case [Eq. (6)] compensates for this decrease in SH such that the surface buoyancy flux does not change much between the dry and the moist BL (Fig. 4). This shows that through the surface buoyancy flux moisture only has a minor effect on the BL structure (although moisture does affect the partitioning between SH and LH).

\section{e. Sensitivities to other parameters}

The conceptual model provides a framework for exploring how the BL structure and the convective mass 
flux depend on other parameters, such as stability, or the dryness of the free troposphere. For the control case, a surface temperature of $301 \mathrm{~K}$ and a humidity inversion jump of $3 \mathrm{~g} \mathrm{~kg}^{-1}$ are prescribed (Table 1). To explore the sensitivity, we either increase $\theta_{\text {sfc }}$ to $302 \mathrm{~K}$, which corresponds to a decrease in stability, or increase $\Delta q$ to $5 \mathrm{~g} \mathrm{~kg}^{-1}$, which corresponds to a drier FT, keeping all other parameters the same (Fig. 4). The sensitivity to $\Delta q$ can be interpreted as a measure for the uncertainty that is introduced by simplifying the cloud-subcloud-layer interactions to keeping $\Delta q$ constant. Changing $\theta_{\text {sfc }}$ or $\Delta q$ leads to the same dependencies of the $\mathrm{BL}$ properties on $Q_{\mathrm{BL}}$ as in the control case. For all variables except $w_{e}$, the moist $\mathrm{BL}$ is less sensitive to an increase in $\theta_{\mathrm{sfc}}$ than the dry BL. The unintuitive behavior of the dry model that for strong radiative $\mathrm{BL}$ cooling $\theta_{\mathrm{BL}}$ decreases with increasing $\theta_{\mathrm{sfc}}$ is not found in the case of the moist $\mathrm{BL}$. For an increase in $\theta_{\text {sfc }}$ with all other parameters (including $Q_{\mathrm{BL}}$ ) fixed, the equations of the dry model enforce a proportionality of $h$ and $\Delta \theta$, which determines $\theta_{\mathrm{BL}}$ (Naumann et al. 2017). In the case of the moist $\mathrm{BL}$ this strict proportionality is broken by the effect of moisture.

An increase in $\Delta q$ by $2 \mathrm{~g} \mathrm{~kg}^{-1}$ or an increase in $\theta_{\text {sfc }}$ by $1 \mathrm{~K}$ affects $w_{e}$ and $w_{m}$ more than a change in radiative BL cooling of several kelvins per day (Fig. 4). Both modifications, $\Delta q$ and $\theta_{\text {sfc }}$, have a similar effect in magnitude on $w_{e}$ and $w_{m}$, although with different sign. For a drier FT $\left(\Delta q=5 \mathrm{~g} \mathrm{~kg}^{-1}\right)$, the BL is drier and hence LH is larger. A larger $\mathrm{LH}$ causes a deeper BL and a stronger $\Delta \theta$. A stronger $\Delta \theta$ reduces $w_{e}[$ Eq. (7)] and hence in equilibrium also $w_{m}$ is smaller in magnitude than in the control case. For a less stable air column $\left(\theta_{\text {sfc }}=302 \mathrm{~K}\right)$, both $\mathrm{SH}$ and LH increase, which cause a deeper BL and higher $\theta_{\mathrm{BL}}$. Because both $h$ and $\theta_{\mathrm{BL}}$ increase, $\Delta \theta$ does not change but $F_{\mathrm{B}}$ increases (due to an increase in $\mathrm{SH}$ and $\mathrm{LH})$. This increase in $F_{\mathrm{B}}$ increases $w_{e}$ [Eq. (7)] and hence in equilibrium also $w_{m}$ is larger in magnitude than in the control case.

The conceptual model also helps us to understand why, in the absence of cloud organization, there are very rarely no clouds in the trades. In the moist model, neither a completely dry FT $\left(q_{\mathrm{FT}}=0\right)$ nor a colder surface $\left(\theta_{\text {sfc }} \rightarrow \theta_{0}\right)$ are able to reduce the convective mass flux to zero (not shown). Testing all prescribed parameters (Table 1), we find that only a very small value of $C_{d} V\left(<0.0011 \mathrm{~m} \mathrm{~s}^{-1}\right)$ or $Q_{\mathrm{FT}} / \Gamma=w_{\mathrm{FT}}$ $\left(<-1.2 \mathrm{~cm} \mathrm{~s}^{-1}\right)$ causes $w_{m}$ to vanish. Both conditions, which correspond to a large-scale wind of $V<1.1 \mathrm{~m} \mathrm{~s}^{-1}$ or a radiative FT cooling rate $Q_{\mathrm{FT}}<-5 \mathrm{Kday}^{-1}$, are unusual in the trades and might explain why it is unlikely to have large areas of clear skies over the ocean in the trades (Nuijens et al. 2014). In the next section, we show how instead a shallow circulation is able to suppress convection in parts of its area of influence resembling patterns of convecting and nonconvecting areas in close vicinity.

\section{Two-column moist conceptual model with a shallow circulation}

Classical mixed-layer models have often been used to study the growth of the mixed layer or the properties of an equilibrium mixed layer without horizontal heterogeneities. In this section we represent a heterogeneity in the form of two neighboring columns, which differ in the prescribed radiative BL cooling or SST. By allowing those two columns to interact via a shallow circulation, we study how the BL equilibrium changes when being coupled dynamically.

\section{a. Formulation of a coupled conceptual model}

To analyze the circulation caused by differences in radiative BL cooling and its effect on the BL properties, we formulate a moist two-column model by analogy with the dry two-column model (Naumann et al. 2017) but based on the moist equations [Eqs. (1)-(10)]. The two columns differ from one another in that the radiative $\mathrm{BL}$ cooling in column $1 Q_{\mathrm{BL}, 1}$ is varied between -2 and $-6 \mathrm{~K} \mathrm{day}^{-1}$ and that the radiative $\mathrm{BL}$ cooling in column $2 Q_{\mathrm{BL}, 2}$ is fixed at $-1 \mathrm{~K}$ day $^{-1}$. This difference in radiative $\mathrm{BL}$ cooling between the two columns causes a pressure difference with colder and denser air in column 1 and drives a BL flow from column 1 to column 2 [Eq. (B11)]. While the BL flow is driven by a pressure difference, the BL flow itself, by advecting air of different density [Eqs. (B1) and (B4)], reduces the pressure difference between the columns and thereby reduces its speed; that is, the flow acts as a negative feedback on itself (Naumann et al. 2017). The BL flow also drives a horizontal return flow, which is imposed to be restricted to the layer between the BL height in column $1 h_{1}$ and in column 2 $h_{2}$ [Eqs. (B12) and (B14)]. Both horizontal flows are connected by vertical motion, which in column 1 adds an additional vertical velocity $w_{s}$ to the balance of $h_{1}$ [Eq. (B7)]. The complete set of equations for the two-column moist conceptual model is given in appendix B [Eqs. (B1)-(B11)]. The equations are identical to Naumann et al. (2017) except for the inclusion of $w_{m}$, the replacements of $\Delta \theta$ and $F_{\theta}$ by $\Delta \theta_{v}$ and $F_{\mathrm{B}}$, respectively, and the formulation of the corresponding moisture equations. Additionally prescribed length scales are chosen to match those of observed 


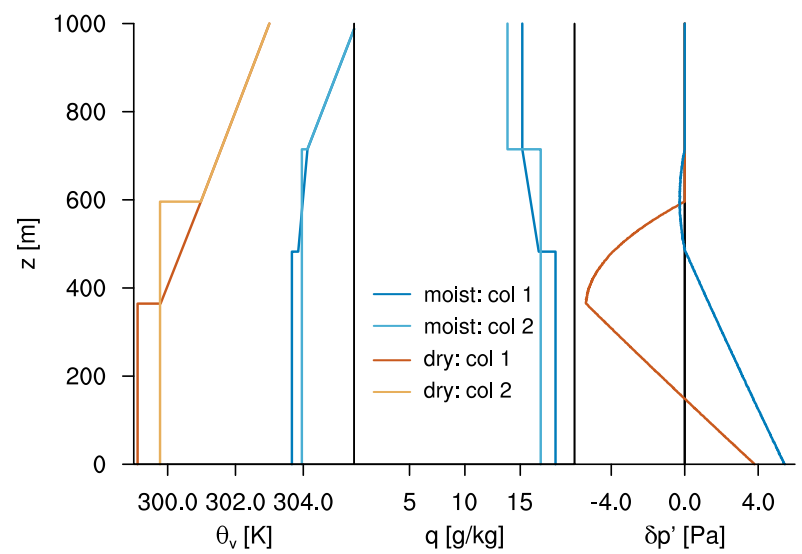

FIG. 5. Equilibrium profiles of the dry and the moist two-column model for $Q_{\mathrm{BL}, 1}=-3 \mathrm{Kday}^{-1}$ and $Q_{\mathrm{BL}, 2}=-1 \mathrm{~K} \mathrm{day}^{-1}$.

mesoscale shallow cumulus organization (Table 1; Bony et al. 2017).

\section{b. Shallow circulations driven by radiative $B L$ cooling differences}

Coupling two radiatively different columns by a pressure-driven circulation causes a BL flow from the cold and moist column (column 1) to the warm and dry column (column 2). This BL flow translates into an additional subsidence term $w_{s}$ in column 1 , which is balanced by stronger $w_{\mathrm{e}, 1}$ [Eq. (B7)]. The additional subsidence due to the shallow circulation decreases the BL height. The decrease in BL height and the stronger entrainment increases $\theta_{\mathrm{BL}, 1}$ in column 1 (Figs. 5, 6; cf. Fig. 3). A warmer BL can, for the same $\mathrm{LCL}$, maintain more moisture; that is, $q_{\mathrm{BL}, 1}$ increases in the coupled setup compared to the homogeneous uncoupled setup. These differences in the BL properties between the coupled and uncoupled setup are rather small in the case of the moist BL compared to the dry BL. In the dry model, $w_{s}$ breaks the strict balance of $w_{e, 1}$ and $w_{\mathrm{FT}}$, which is already broken by $w_{m}$ in the moist case. In the coupled dry model, $w_{s}$ decreases $h_{1}$ and $w_{e, 1}$ is considerably stronger to balance $w_{s}$ compared to the uncoupled setup. Physically, the smaller $\Delta \theta$ in the coupled dry setup allows for updrafts to penetrate the inversion more easily and hence $w_{e}$ is larger than in the uncoupled case. Overall, the BL properties of the dry and the moist case are more alike if a shallow circulation is included (Fig. 6; cf. Fig. 4). The more alike BL properties already hint at a
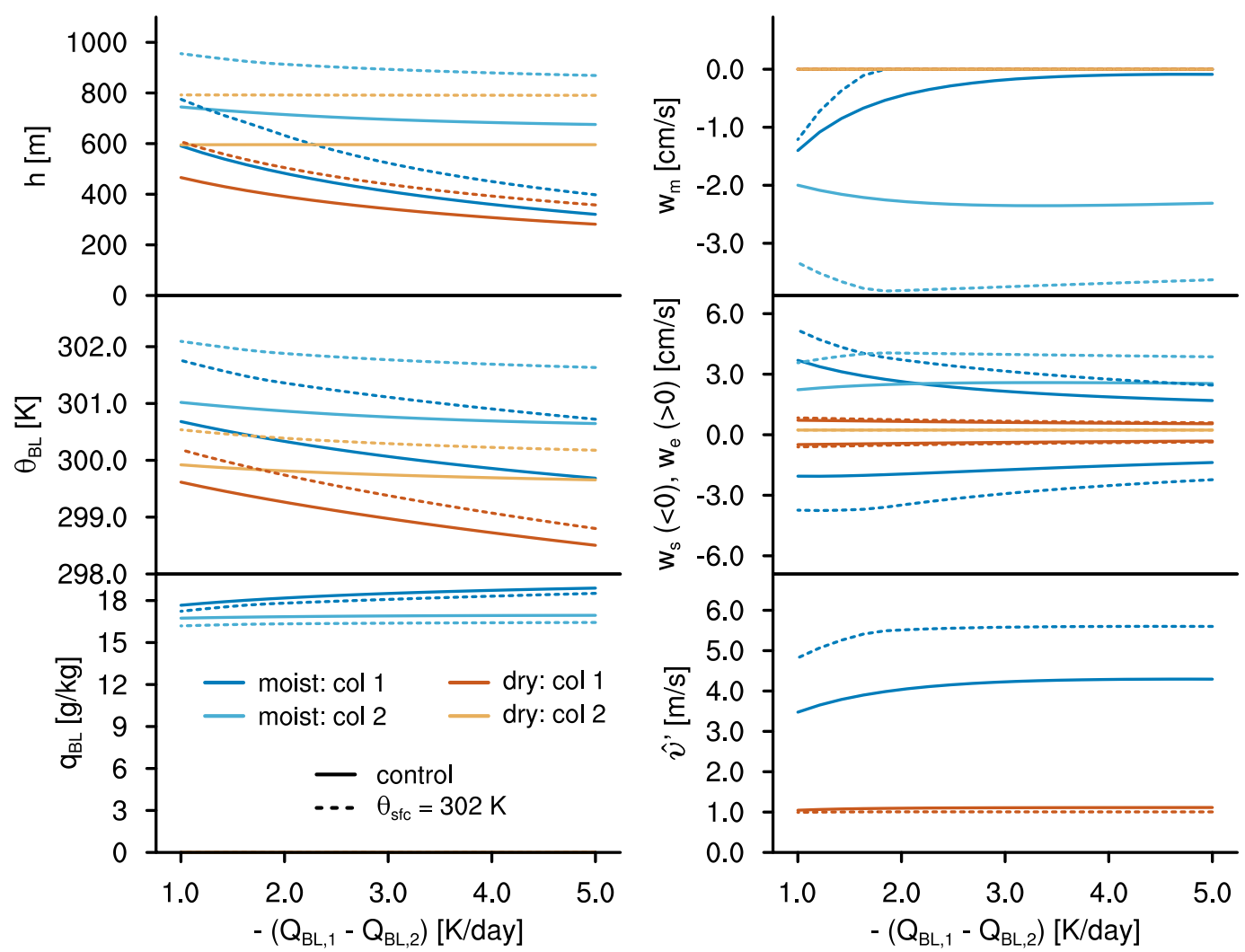

FIG. 6. Equilibrium of the dry and the moist two-column model for $Q_{\mathrm{BL}, 1}=-2$ to $-6 \mathrm{~K}_{\text {day }}{ }^{-1}$ and $Q_{\mathrm{BL}, 2}=-1 \mathrm{~K}$ day $^{-1}$. For the control case, $\theta_{\mathrm{sfc}}=301.0 \mathrm{~K}$. 
stronger influence of the shallow circulation (which is present in the dry case and in the moist case) than of the convective mass flux.

In the moist two-column model, the pressure difference $\delta p^{\prime}$ and hence the BL flow $\hat{v}^{\prime}$, which depends on $\delta p^{\prime}$ integrated over the BL height [Eq. (B11)], are considerably stronger than in the dry two-column model $\left(\hat{v}^{\prime}=4 \mathrm{~m} \mathrm{~s}^{-1}\right.$ in the moist case; $\hat{v}^{\prime}=1 \mathrm{~m} \mathrm{~s}^{-1}$ in the dry case; Figs. 5, 6). The magnitude of this effect is moderated by two mechanisms: First, while the difference in $\theta_{\mathrm{BL}}$ between the columns is similar in the dry and in the moist case, the difference in $\theta_{v, \mathrm{BL}}$ is smaller in the moist case because the colder column 1 is also moister. Due to the smaller difference in $\theta_{v, \mathrm{BL}}$ in the case of the moist $\mathrm{BL}$, the increase in $\delta p^{\prime}$ with decreasing height is smaller than in the case of the dry BL (Fig. 5). Hence the virtual effect of the BL moisture would result in a smaller $\delta p^{\prime}$ in the BL and a slower BL flow in the moist case, in contrast to what is found in Fig. 6.

The virtual effect is, however, overcompensated by a second contribution to $\delta p^{\prime}$ that is rooted in the structure of the return flow layer: $\Delta \theta_{v}$ is considerably smaller in both columns in the moist case compared to the dry case and the return flow layer is less cooled by the advection of $\mathrm{BL}$ air from column 2 in the moist case as compared to the dry case. Therefore, at the height of the return flow layer, the temperature difference between column 1 and column 2 is small and hence the negative pressure bulge is small in the moist case (Fig. 5). While in the moist case $\delta p^{\prime}$ near the height of $h_{1}$ is close to zero $\left(h_{1} \approx 500 \mathrm{~m}\right.$ in Fig. 5), $\delta p^{\prime}$ amounts to about $-5 \mathrm{~Pa}$ at $h_{1}$ in the dry case $\left(h_{1} \approx 400 \mathrm{~m}\right)$. Therefore, although the increase in $\delta p^{\prime}$ toward the surface is stronger than in the moist case, the less negative value of $\delta p^{\prime}$ at $h_{1}$ in the moist case results in a larger positive value of $\delta p^{\prime}$ integrated over the BL and hence a stronger BL flow.

As in the dry case, also in the moist case, the strength of the $\mathrm{BL}$ flow is insensitive to stronger radiative $\mathrm{BL}$ cooling differences above a threshold (Fig. 6). In the moist BL, a BL flow of $3.5 \mathrm{~m} \mathrm{~s}^{-1}$ develops for a radiative cooling difference as small as $1 \mathrm{~K} \mathrm{day}^{-1}$ and the BL flow saturates at $4.0 \mathrm{~m} \mathrm{~s}^{-1}$ for a radiative cooling difference of $2 \mathrm{~K} \mathrm{day}^{-1}$ and more. For a stronger radiative BL cooling difference, $h_{2}$ decreases slightly, $h_{1}$ decreases more strongly, and the difference between $\theta_{\mathrm{BL}, 1}$ and $\theta_{\mathrm{BL}, 2}$ increases. On the one hand, for a decrease in $h_{2}$ and $h_{1}$, the height of $\delta p^{\prime}=0$ decreases; that is, positive $\delta p^{\prime}$ builds up over a shallower layer. One the other hand, the larger the difference between $\theta_{\mathrm{BL}, 1}$ and $\theta_{\mathrm{BL}, 2}$ the stronger the increase in $\delta p^{\prime}$ with decreasing height. For an increase in radiative $\mathrm{BL}$ cooling difference, both effects compensate and hence the strength of the BL flow is insensitive to the difference in radiative BL cooling.
For the moist case, $w_{m}$ is similar in both columns if similar values of radiative BL cooling are applied but decreases rapidly in column 1 when stronger differences in radiative $\mathrm{BL}$ cooling are prescribed. In the prognostic equation for $h_{1}$ [Eq. (B7)], the positive tendency of $w_{e}$ is balanced by $w_{m}, w_{\mathrm{FT}}$, and $w_{s}$. Independently of the applied radiative BL cooling, the vertical velocity contribution from large-scale subsidence $w_{\mathrm{FT}}\left(=-0.23 \mathrm{~cm} \mathrm{~s}^{-1}\right)$ is about an order of magnitude weaker than the other contributions. With stronger radiative $\mathrm{BL}$ cooling in column $1, w_{\mathrm{m}, 1}$ decreases and $w_{\mathrm{e}, 1}$ is essentially balanced by $w_{s}$. This means that the shallow circulation reduces $h_{1}$ and increases $\theta_{\mathrm{BL}, 1}$ such that the LCL does not fall below $h_{1}$ anymore and therefore convection is suppressed. In column 1 the shallow circulation is hence more efficient in controlling $h_{1}$ than is convection. In column 2 , the shallow circulation does not directly influence $h_{2}$. Also, $w_{\mathrm{m}, 2}$, which plays an essential role in column 2 independent of the applied radiative $\mathrm{BL}$ cooling in column 1 , is about twice as large as in the homogeneous case and agrees well in magnitude with a modeling study by Bretherton and Blossey (2017). This shows how a radiatively driven shallow circulation is able to suppress convective ventilation in areas of strong radiative $\mathrm{BL}$ cooling and enhance convection in areas of weak radiative BL cooling.

Increasing $\theta_{\text {sfc }}$ by $1 \mathrm{~K}$ to $302 \mathrm{~K}$, that is, decreasing the stability of the column, increases all velocities $\left(\hat{v}^{\prime}, w_{s}, w_{e}\right.$, and $w_{m}$ ) in the moist case substantially (Fig. 6). The increase in $\hat{v}^{\prime}$ is caused by an increase in $h$ with higher $\theta_{\text {sfc }}$. With the same difference in $\theta_{v, \text { BL }}$ between the columns, the pressure difference can build up over a deeper BL. Therefore, $\delta p^{\prime}$ reaches higher values in the BL and $\hat{v}^{\prime}$ is stronger for higher $\theta_{\text {sfc }}$. Because $w_{s}$ is directly proportional to $\hat{v}^{\prime}$ [Eq. (B7)], it is also stronger for higher $\theta_{\text {sfc }}$. A higher $\theta_{\text {sfc }}$ also causes a higher latent heat flux at the surface, both because $q_{\mathrm{sfc}}$ is higher and because $q_{\mathrm{BL}}$ is lower (due to higher $h$; see section 2e). This higher latent heat flux for higher $\theta_{\text {sfc }}$ results in a higher buoyancy flux [Eq. (B9)] and therefore in a higher $w_{e}$ [Eq. (B7)]. In column $1, w_{s}$ and $w_{e, 1}$ balance and $w_{m, 1}$ is suppressed (except for weak radiative BL cooling). In column 2, a stronger $w_{e, 2}$ for higher $\theta_{\text {sfc }}$ is balanced by an enhanced $w_{m, 2}$. The sensitivity to increasing $\Delta q$ by $2 \mathrm{~g} \mathrm{~kg}^{-1}$ or decreasing $V$ by $2 \mathrm{~m} \mathrm{~s}^{-1}$ (not shown) is smaller than the sensitivity to increasing $\theta_{\text {sfc }}$ by $1 \mathrm{~K}$. Both modifications suppress $w_{m, 1}$ for smaller radiative cooling differences than the control case.

\section{c. Shallow circulations driven by SST differences}

Instead of being driven by a horizontal difference in radiative $\mathrm{BL}$ cooling, a shallow circulation can also 

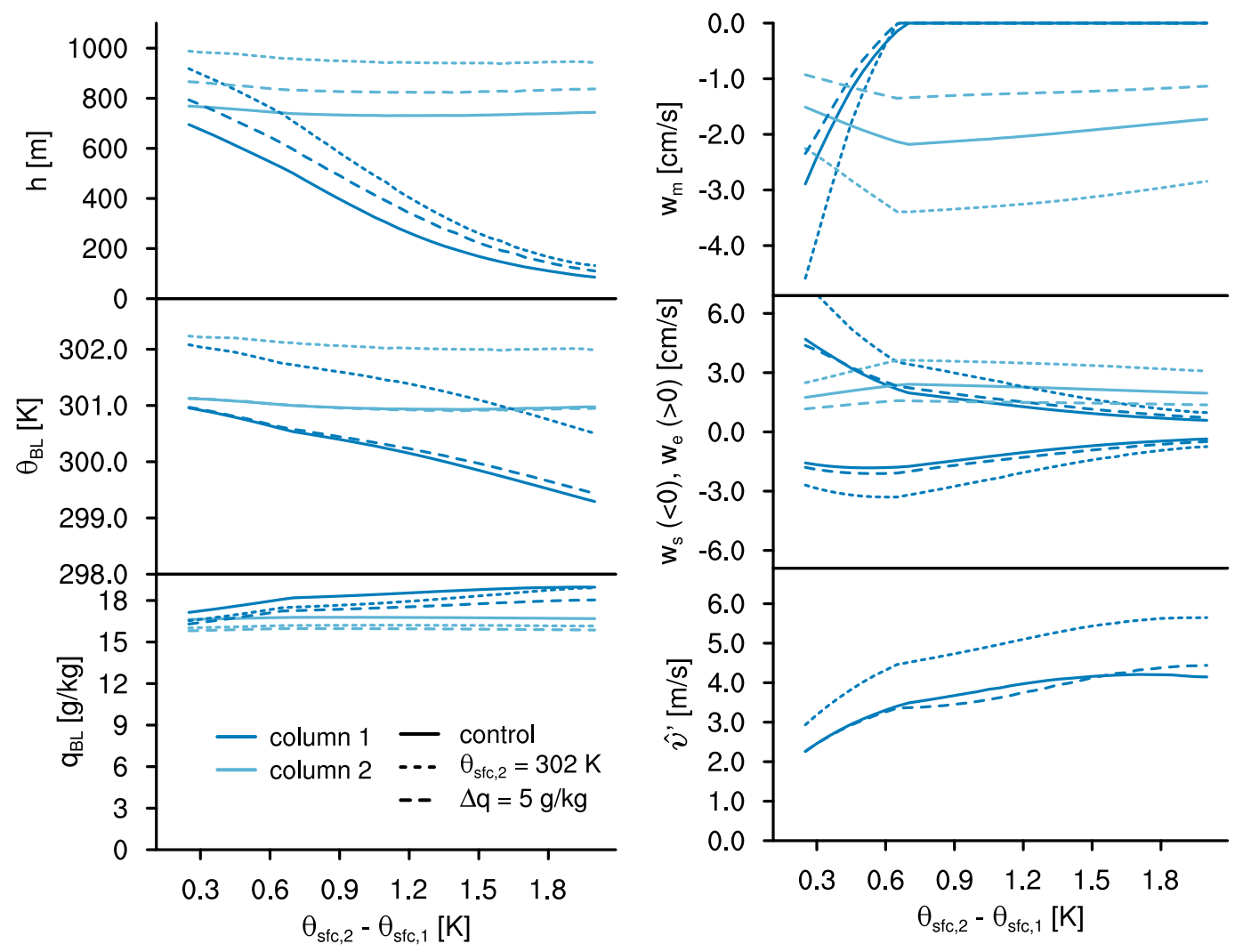

FIG. 7. Equilibrium of the moist two-column model with homogeneous radiative cooling $\left(Q_{\mathrm{FT}}=Q_{\mathrm{BL}, 1}=\right.$ $\left.Q_{\mathrm{BL}, 2}=-1.0 \mathrm{~K} \mathrm{day}^{-1}\right)$ and heterogeneous surface temperature: $Q_{\mathrm{sfc}, 2}-Q_{\mathrm{sfc}, 1}=0.25-2.0 \mathrm{~K}$. For the control case $\theta_{\mathrm{sfc}, 2}=301.0 \mathrm{~K}$ and $\Delta q=3 \mathrm{~g} \mathrm{~kg}^{-1}$.

be caused by a horizontal difference in SST. Radiative cooling and SST anomalies are likely coupled; that is, they reinforce each other. By prescribing a homogeneous radiative cooling (i.e., $Q_{\mathrm{BL}, 1}=Q_{\mathrm{BL}, 2}=$ $\left.Q_{\mathrm{FT}}=-1 \mathrm{~K} \mathrm{day}^{-1}\right)$ and a difference in surface temperature between column 1 and $2\left(\theta_{\text {sfc }, 1}=299.0-300.75 \mathrm{~K}\right.$ and $\theta_{\mathrm{sfc}, 2}=301.0 \mathrm{~K}$ ), we separate the effects of SST differences from radiative effects in the coupled twocolumn model. We find that a typical SST difference of $0.6 \mathrm{~K}$ drives a BL flow of $3.3 \mathrm{~m} \mathrm{~s}^{-1}$ (Fig. 7), which is somewhat less strong than a BL flow driven by a typical radiative $\mathrm{BL}$ cooling difference of $3 \mathrm{~K}$ day $^{-1}$.

Differences between an SST-driven shallow circulation and a radiatively driven shallow circulation in terms of their BL properties are overall small and the same physical mechanisms apply (cf. Figs. 6 and 7; section 3b): with increasing difference in SST or with increasing radiative $\mathrm{BL}$ cooling difference, the difference between the two columns in terms of $h, \theta_{\mathrm{BL}}$, and $q_{\mathrm{BL}}$ increases and in column $1 w_{m, 1}$ becomes zero above a certain value of SST difference or radiative BL cooling difference.

A difference is found for the dependence of $\hat{v}^{\prime}$ on the prescribed SST or radiative BL cooling gradient: $\hat{v}^{\prime}$ increases with increasing difference in SST but quickly saturates in magnitude to an increase in radiative $\mathrm{BL}$ cooling difference. This distinct behavior of $\hat{v}^{\prime}$ can be explained as follows: While in the radiatively driven two-column model $h_{2}$ decreases slightly, $h_{2}$ is insensitive to increasing SST difference. A constant $h_{2}$ and a decrease in $h_{1}$ for larger surface temperature difference allows the height of $\delta p^{\prime}=0$ to decrease slightly; that is, $\delta p^{\prime}$ builds up over a slightly thinner layer. At the same time, the difference between $\theta_{\mathrm{BL}, 1}$ and $\theta_{\mathrm{BL}, 2}$ increases with increasing surface temperature difference more strongly than for increasing radiative BL cooling difference. The stronger difference between $\theta_{\mathrm{BL}, 1}$ and $\theta_{\mathrm{BL}, 2}$ causes a stronger increase in $\delta p^{\prime}$ with decreasing height. While both effects balance for a radiatively driven BL flow, for an SST-driven BL flow the second effects dominates and leads to an increase in $\delta p^{\prime}$ and therefore to an increase in $\hat{v}^{\prime}$ for a larger surface temperature difference. The effect of the increase in $\hat{v}^{\prime}$ on $w_{s}$ is overcompensated by the strong decrease of $h_{1}$ so that $w_{s}=-\hat{v}^{\prime} h_{1} / X_{1}$ decreases with higher surface temperature difference. Because $w_{m, 1}$ is suppressed for strong surface temperature differences, 
$w_{e, 1}$ follows $w_{s}$ and decreases for stronger surface temperature differences.

Another difference to the radiatively driven twocolumn model is that for low $\theta_{\mathrm{sfc}, 1}, \theta_{\mathrm{sfc}, 1}<\theta_{\mathrm{BL}, 1}$; that is, $F_{\theta, 1}$ becomes negative (up to $\mathrm{SH}=-3 \mathrm{~W} \mathrm{~m}^{-2}$ for an SST difference of $2.0 \mathrm{~K}$ ) and the $\mathrm{BL}$ is losing heat to the surface. In terms of the heat balance, entrainment warming in column 1 is then balanced by radiative cooling and cooling from the surface. Because $F_{q, 1}$ is positive, $F_{B}$ remains positive in both columns.

Similar to the radiatively driven model, the SSTdriven two-column model is not sensitive to an increase in the moisture inversion strength (Fig. 7). For a stronger moisture inversion strength, the BL is somewhat deeper but the strength of the BL flow and all other variables are almost unchanged. In analogy with the radiatively driven model, an increase in the SST in both columns (retaining the SST difference between column 1 and 2) increases the strength of the BL flow also in the SST-driven two-column model.

\section{Conclusions}

In this study, we analyze how moisture and convection modify the BL structure and a shallow circulation driven by differential radiative BL cooling or SST gradients. For this purpose, we extend a dry conceptual model (Naumann et al. 2017) to include a prognostic equation for BL moisture and a simple formulation of convective mass flux that is based on the LCL and ventilates the BL.

In the homogeneous, uncoupled setup of this conceptual model [Eqs. (1)-(10)], we are interested in the equilibrium of the BL, its difference to the dry case, and how this equilibrium changes if the prescribed radiative cooling rates are modified. A linearized solution of the system shows an insensitivity of the BL water vapor mixing ratio and inversion entrainment to radiative BL cooling. This insensitivity arises from a reduction of the $\mathrm{BL}$ temperature in response to stronger radiative $\mathrm{BL}$ cooling, which requires a shallowing of the $\mathrm{BL}$ as the LCL lowers with cooling. Thereby, convection acts as a moisture valve to the $\mathrm{BL}$ varying the $\mathrm{BL}$ height while keeping $\mathrm{BL}$ water vapor mixing ratio roughly constant. By this mechanism the conceptual model can explain the observed range of BL height and near-surface relative humidity in the trades, which vary moderately between 400 and $1000 \mathrm{~m}$ and $70 \%-90 \%$, respectively (O. Tessiot 2018, personal communication; Lonitz et al. 2015; Klingebiel et al. 2019).

Compared to a completely dry BL, moisture leads to a warming of the $\mathrm{BL}$ and a weakening of the inversion jump. Both of these features can be explained by the convective mass flux in the moist case, which enhances the entrainment at the inversion. The enhanced entrainment warms the BL and generates the need for a small inversion strength in order for the BL temperature to stabilize. In this way the convective mass flux dictates the behavior of the BL.

To analyze how a shallow circulation that is driven by differential radiative cooling is modified by moisture, we formulate a two-column model [Eqs. (B1)-(B11)]. By applying a stronger radiative BL cooling in one column than in the other column, a pressure difference develops between the columns, which drives a BL flow from the cold and moist to the warm and dry column and couples the columns dynamically through a shallow circulation. A BL flow of $3.5 \mathrm{~m} \mathrm{~s}^{-1}$ develops for a radiative cooling difference of $1 \mathrm{Kday}^{-1}$ and the BL flow saturates at $4.0 \mathrm{~m} \mathrm{~s}^{-1}$ for a radiative cooling difference of $2 \mathrm{~K} \mathrm{day}^{-1}$ and more. The pressure difference and hence the BL flow is stronger in the moist case than in the dry case $\left(\hat{v}^{\prime}=1 \mathrm{~m} \mathrm{~s}^{-1}\right)$ because the inversion jump is weaker in the moist case, which effectively confines the pressure gradient to the subcloud layer and prevents a negative pressure bulge near the inversion height. This inversion effect overcompensates the virtual effect of BL moisture, which works to reduce the difference in virtual potential temperature in the BL and hence the difference in pressure between the columns.

The moist BL reacts in a similar way to a shallow circulation driven by differential radiative BL cooling as it behaves for a shallow circulation driven by differential SST. Unlike the strength of the shallow circulation driven by differential radiative BL cooling, which is independent of an increase in radiative BL cooling difference, the strength of the shallow circulation driven by differential SST decreases with decreasing SST difference. Over the subsidence-dominated shallow convective areas of the tropical Atlantic typical gradients in SST are around $0.6 \mathrm{~K}(200 \mathrm{~km})^{-1}$ (Fig. 1). In the moist conceptual model such an SST gradient results in a BL flow of $\hat{v}^{\prime}=3.3 \mathrm{~m} \mathrm{~s}^{-1}$, which is weaker than the BL flow caused by a typical gradient in radiative BL cooling of $3 \mathrm{~K} \mathrm{day}^{-1}(200 \mathrm{~km})^{-1}\left(\hat{v}^{\prime}=4.0 \mathrm{~m} \mathrm{~s}^{-1}\right)$. We therefore conclude that besides SST gradients or land-ocean contrasts that induce a sea breeze, also spatial differences in radiative BL cooling should be considered as a possible mechanism for the formation of shallowand perhaps eventually deep-circulations. For typical conditions in the trades, radiative gradients might potentially drive stronger shallow circulations than SST gradients.

To reduce the degrees of freedom, the conceptual model assumes a fixed humidity inversion strength; that is, the humidity inversion strength is not coupled, for 
example, to the convective mass flux. In the two-column model more radiative $\mathrm{BL}$ cooling decreases the convective mass flux. We speculate that less convective mass flux out of the BL leads to more drying aloft and hence increases radiative cooling at the BL top. Likewise the warmer column sees more convective mass flux, which would increase moisture aloft and lead to less radiative cooling. If cloud radiative effects are small compared to the radiative effect of moistening and drying above the cloud layer, one might expect these dynamics to be self-amplifying. Another mechanism for self-amplification could be the onset of precipitation in the warmer column, which might heat the cloud layer and drive adjacent subsidence in the colder column. Although this study is limited to shallow convection the buildup of moisture and increased convergence within the warm column could, through the self-amplification mechanisms, also help precondition deep convection.

In the conceptual model the velocity balance at the BL top is quite different between the dry and the moist case as well as between an uncoupled and a coupled setup: In the homogeneous uncoupled dry case, the entrainment velocity at the inversion is strictly balanced by large-scale subsidence, which is prescribed by the FT temperature gradient and the FT radiative cooling $\left(w_{\mathrm{FT}}=-0.23 \mathrm{~cm} \mathrm{~s}^{-1}\right)$. Including moisture in the homogeneous case, the entrainment velocity is substantially larger than in the dry case and balances the convective mass flux velocity $\left(\approx-1 \mathrm{~cm} \mathrm{~s}^{-1}\right)$ while the large-scale subsidence plays a minor role. The convective mass flux velocity is insensitive to the applied radiative BL cooling but increases with a weaker stability or a wetter FT. In the uncoupled moist conceptual model an insignificant convective mass flux can only be reached with prescribing unusually low large-scale surface winds or unusually strong large-scale subsidence. This might explain why, in the absence of convective organization, it is very unlikely to have vast areas of cloud-free conditions over the tropical oceans.

In the coupled two-column setup the shallow circulation effectively suppresses convection in the descending part of the shallow circulation. Independent of whether moisture is considered or not, the main balance in the descending part is between the shallow circulation and the entrainment velocity. Hence, the shallow circulation is more efficient in controlling the $\mathrm{BL}$ height than is convection. In the ascending part of the shallow circulation the convective mass flux is about twice as strong as in the uncoupled moist case and the balance is between the convective mass flux and the entrainment velocity. Therefore the dynamical coupling between two areas with different radiative BL cooling or SST significantly modifies the BL balance and its properties, a mechanism that is not considered in classical uncoupled bulk conceptual models. In particular, the shallow circulation is able to suppress convection in colder areas and enhance convection in warmer areas. We hypothesize that over the subsidence-dominated tropical oceans this mechanism resembles patterns of convecting and nonconvecting areas in close vicinity to each other, that is, structures of organized shallow convection such as bands with alternating shallow convective and cloud-free areas or mesoscale patches of shallow convection surrounded by cloud-free areas. The model also suggests that shallow organization is as much marked by its cloud-free as by its cloudy properties. Compared to the uncoupled model, the average convective mass flux of two coupled columns is somewhat higher for the same average radiative BL cooling. This implies that besides the importance of heterogeneities for the spatial distribution of the convective mass flux, heterogeneities in connection with a shallow circulation can also increase the average convective mass flux or cloudiness in a region.

Acknowledgments. We thank Julia Windmiller, Alan Betts, and two anonymous reviewers for their helpful comments on the manuscript and ICDC for providing the Reynolds SST dataset. Primary data and scripts used in the analysis and other supplementary information that may be useful in reproducing the author's work are archived by the Max Planck Institute for Meteorology and can be obtained by contacting publications@ mpimet.mpg.de. This research was carried out as part of the Hans Ertel Centre for Weather Research. This research network of universities, research institutes, and the Deutscher Wetterdienst is funded by the Federal Ministry of Transport and Digital Infrastructure (BMVI).

\section{APPENDIX A}

\section{Transient Response of the Homogeneous Model}

For the transient response, we use the case of the dry BL as the initial state and solve Eqs. (1)-(10) including the moist components. The moistening time scale of the BL until a new equilibrium is reached is about 3-4 days. Once convections sets in, the adjustment time to equilibrium is about 1 day, which is consistent with Bellon and Stevens (2013). The change in BL height is determined by a balance of $w_{e}, w_{m}$, and $w_{\mathrm{FT}}$ [Eq. (7)]. When the $\mathrm{BL}$ is moistening from its initial dry state, $w_{e}$ dominates and the $\mathrm{BL}$ deepens until saturation is reached (Fig. A1; after 1-2 days). After saturation is reached, $w_{m}$ is the dominant term in Eq. (7) and $h$ 

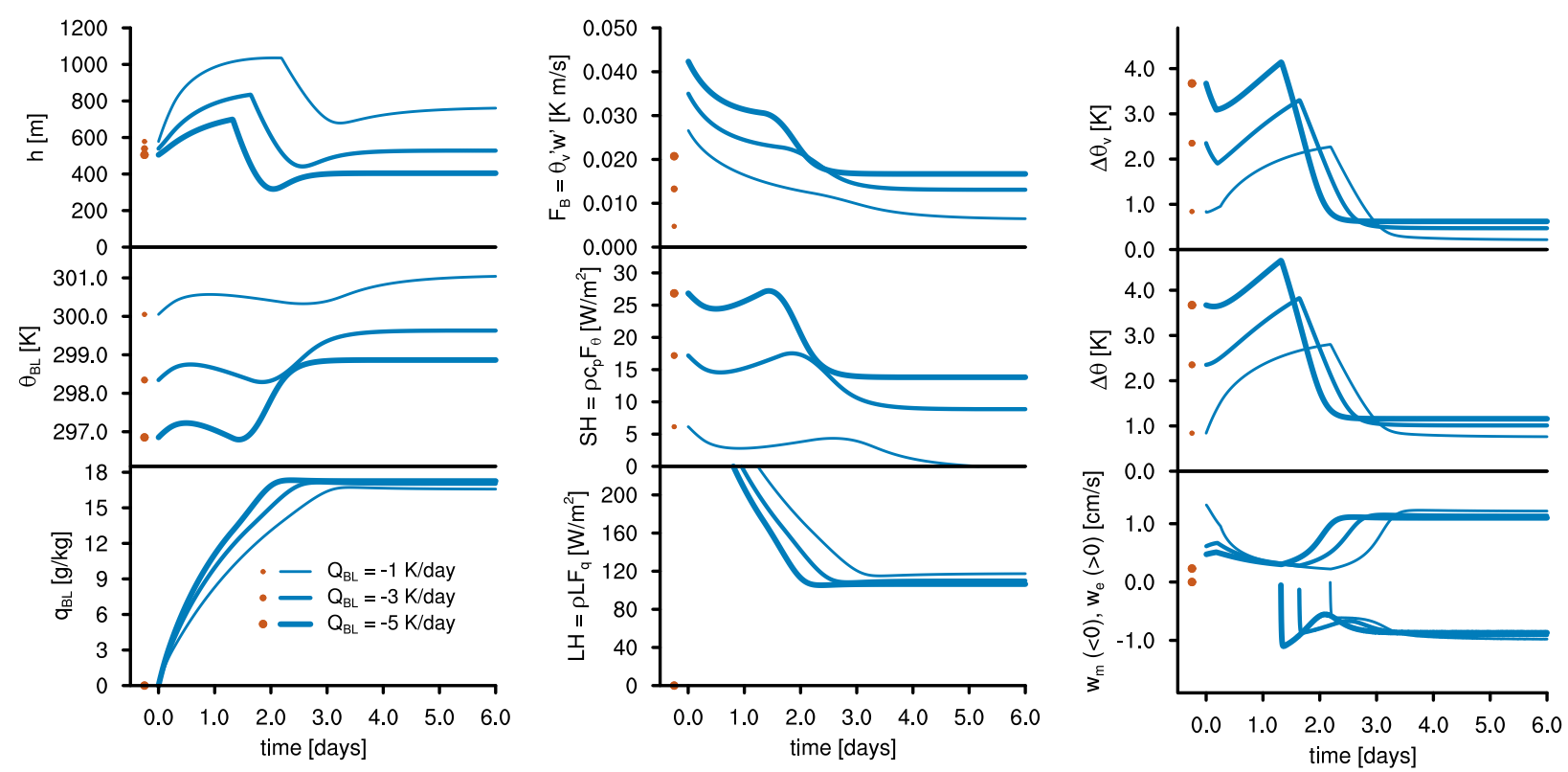

FIG. A1. Temporal evolution of the one-column model from the dry case to the moist equilibrium case. The moist temporal evolution is in blue, and the dry initial conditions are in brown.

decreases until a balance of all three terms determines the equilibrium. For strong radiative $\mathrm{BL}$ cooling, $\theta_{\mathrm{BL}}$ is lower and the inversion jump $\Delta \theta_{v}$ is larger. Therefore, in the initial $w_{e}$-dominated period $w_{e}$ is weaker for strong radiative $\mathrm{BL}$ cooling and the $\mathrm{BL}$ remains shallower than for weak radiative $\mathrm{BL}$ cooling.

The moist equilibrium BL is warmer than the dry equilibrium $\mathrm{BL}$ and more pronounced for strong radiative BL cooling. The transient evolution from the dry to the moist equilibrium shows that $\theta_{\mathrm{BL}}$ increases mostly in the saturation phase, when $w_{m}$ dominates and $h$ decreases (Fig. A1). Besides the adjustment of $\Delta \theta$ to the moist $\mathrm{BL}$, which in the transient response allows for strong entrainment warming, also the decrease in $h$ makes the entrainment warming more effective as the heating is distributed over a shallower BL.

\section{APPENDIX B}

\section{Formulation of a Moist Two-Column Model with a Shallow Circulation}

For column $i \in[1,2]$,

$$
\begin{aligned}
\frac{\partial \theta_{\mathrm{BL}, i}}{\partial t}= & Q_{\mathrm{BL}, i}+\frac{1}{h_{i}}\left(w_{e, i} \Delta \theta_{i}+F_{\theta, i}\right) \\
& +\delta_{2, i}\left(\hat{\boldsymbol{v}}^{\prime} \frac{\theta_{\mathrm{BL}, 1}-\theta_{\mathrm{BL}, 2}}{X_{2}} \frac{h_{1}}{h_{2}}\right),
\end{aligned}
$$

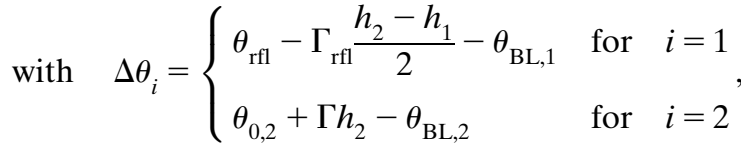

$$
F_{\theta, i}=C_{d}\left(V+0.5 \hat{v}^{\prime}\right)\left(\theta_{\mathrm{sc}, i}-\theta_{\mathrm{BL}, i}\right)
$$

$$
\frac{\partial q_{\mathrm{BL}, i}}{\partial t}=\frac{1}{h_{i}}\left(w_{e, i} \Delta q_{i}+F_{q, i}\right)+\delta_{2, i}\left(\hat{\boldsymbol{v}}^{\prime} \frac{q_{\mathrm{BL}, 1}-q_{\mathrm{BL}, 2}}{X_{2}} \frac{h_{1}}{h_{2}}\right)
$$

with

$$
\Delta q_{i}=\left\{\begin{array}{lll}
q_{\mathrm{rfl}}-\Gamma_{\mathrm{rfl}, q} \frac{h_{2}-h_{1}}{2}-q_{\mathrm{BL}, 1} & \text { for } & i=1 \\
q_{\mathrm{FT}, 2}-q_{\mathrm{BL}, 2} & \text { for } & i=2
\end{array},\right.
$$

$$
F_{q, i}=C_{d}\left(V+0.5 \hat{v}^{\prime}\right)\left(q_{\mathrm{sfc}, i}-q_{\mathrm{BL}, i}\right) ; \quad \text { and }
$$

$$
\begin{aligned}
\frac{\partial h_{i}}{\partial t} & =w_{\mathrm{FT}}+w_{e, i}+\delta_{1, i} w_{s}+w_{m, i} \\
& =\frac{Q_{\mathrm{FT}}}{\Gamma}+A \frac{F_{\mathrm{B}, i}}{\Delta \theta_{v, i}}-\delta_{1, i} \hat{v}^{\prime} \frac{h_{1}}{X_{1}}+w_{m, i},
\end{aligned}
$$




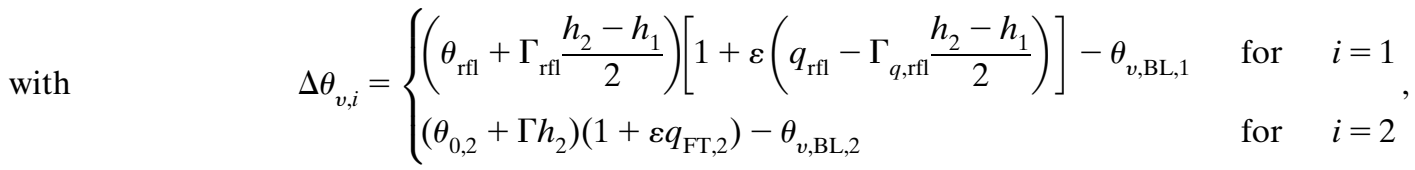

$$
\begin{aligned}
F_{\mathrm{B}, i} & =F_{\theta, i}+\varepsilon \theta_{\mathrm{BL}, i} F_{q, i}, \\
w_{m, i} & =\left\{\begin{array}{lll}
-\frac{h_{i}-\mathrm{LCL}_{i}}{\tau} & \text { if } & \mathrm{LCL}_{i}<h_{i}, \\
0 & \text { if } & \mathrm{LCL}_{i} \geq h_{i}
\end{array}\right.
\end{aligned}
$$

where the BL flow $\hat{v}^{\prime}$ is driven by the pressure difference between column 1 and 2 integrated over the $\mathrm{BL} \delta \hat{p}^{\prime}$ and depends on the spatial scale of the pressure gradient $X_{p}$, which is a prescribed parameter (Table 1). To calculate $\delta \hat{p}^{\prime}$ we integrate over the $\theta_{v}$ profile with positive $\delta p^{\prime}$ and prescribe a pressure of $850 \mathrm{hPa}$ at $1500 \mathrm{~m}$ for both columns (section 3a in Naumann et al. 2017). The formulation of $\hat{v}^{\prime}$ follows Naumann et al. [2017, Eq. (25) therein]:

$$
\hat{v}^{\prime}=-V+\sqrt{V^{2}+\frac{1}{C_{d} \rho} \frac{h_{1}}{X_{p}} \delta \hat{p}^{\prime}} .
$$

In the budget equation for $h_{1}$ [Eq. (B7)], the shallow circulation decreases $h_{1}$, which is represented in $w_{s}$. Additional prescribed parameters are the horizontal scale of column 1 and $2, X_{1}$ and $X_{2}$, respectively (Table 1).

The return-flow layer (index "rfl") is situated on top of the BL in column 1 , is characterized by gradients in potential temperature $\Gamma_{\text {rfl }}$ and humidity $\Gamma_{q, \text { rfl }}$, and its height is defined by $h_{2}-h_{1}$ (see Fig. 9 of Naumann et al. 2017). We formulate two budget equations for $\theta_{\mathrm{rfl}}$ and $q_{\mathrm{rfl}}$ :

$$
\frac{\partial \theta_{\mathrm{rfl}}}{\partial t}=Q_{\mathrm{FT}}-\left(w_{\mathrm{FT}, 1}+0.5 w_{s}\right) \Gamma_{\mathrm{rfl}}+\hat{v}^{\prime} \frac{h_{1}}{h_{2}-h_{1}} \frac{\theta_{\mathrm{BL}, 2}-\theta_{\mathrm{rfl}}}{X_{1}}
$$

with

$$
\begin{aligned}
\Gamma_{\mathrm{rfl}} & =\frac{\theta_{0,1}+\Gamma h_{2}-\theta_{\mathrm{rfl}}}{0.5\left(h_{2}-h_{1}\right)}, \quad \text { and } \\
\frac{\partial q_{\mathrm{rfl}}}{\partial t} & =-\left(w_{\mathrm{FT}, 1}+0.5 w_{s}\right) \Gamma_{q, \mathrm{rfl}}+\hat{\boldsymbol{v}}^{\prime} \frac{h_{1}}{h_{2}-h_{1}} \frac{q_{\mathrm{BL}, 2}-q_{\mathrm{rfl}}}{X_{1}},
\end{aligned}
$$

with

$$
\Gamma_{q, \mathrm{rfl}}=\frac{q_{\mathrm{FT}, 1}-q_{\mathrm{rfl}}}{0.5\left(h_{2}-h_{1}\right)} .
$$

To satisfy the weak temperature gradient assumption, we assume that the FT virtual potential temperature profile in column 1 is set by column 2 : $\theta_{0,1}=\theta_{0,2}\left(1+\varepsilon q_{\mathrm{FT}, 2}\right) /\left(1+\varepsilon q_{\mathrm{FT}, 1}\right)$. This compensates for density differences caused by differences in $q_{\mathrm{FT}}$ between the columns.

If $h_{1}$ approaches $h_{2}$, the flow velocity in the return flow layer, $\hat{v}^{\prime} h_{1} /\left(h_{2}-h_{1}\right)$, becomes large and the solution of the two-column model may become unstable. This is not an issue for the equilibrium solutions in the range of parameters shown in this study but can be an issue during the integration of the prognostic equations depending on the initial conditions. To avoid this issue, we define a minimum return flow-layer height, $h_{\mathrm{rfl}, \min }=25 \mathrm{~m}$ below which we assume that the shallow circulation is not closed in its upper branch but that instead the BL flow increases $h_{2}$. In this case, the equations above are modified by adding the term $\delta_{2, i} w_{s, 2}=\delta_{2, i} \hat{v}^{\prime} h_{1} / X_{2}$ on the right-hand side of Eq. (B7) and by omitting the advection terms in the return flow layer [rightmost term on the right-hand side of Eqs. (B12) and (B14)].

\section{REFERENCES}

Albrecht, B. A., A. K. Betts, W. H. Schubert, and S. K. Cox, 1979: Model of the thermodynamic structure of the trade-wind boundary layer: Part I. Theoretical formulation and sensitivity tests. J. Atmos. Sci., 36, 73-89, https://doi.org/10.1175/ 1520-0469(1979)036<0073:MOTTSO>2.0.CO;2.

Back, L. E., and C. S. Bretherton, 2009: On the relationship between SST gradients, boundary layer winds, and convergence over the tropical oceans. J. Climate, 22, 4182-4196, https://doi.org/10.1175/2009JCLI2392.1.

Bellon, G., and B. Stevens, 2013: Time scales of the trade wind boundary layer adjustment. J. Atmos. Sci., 70, 1071-1083, https://doi.org/10.1175/JAS-D-12-0219.1.

_- O. Reitebuch, and A. K. Naumann, 2017: Shallow circulations: Relevance and strategies for satellite observation. Surv. Geophys., 38, 1509-1528, https://doi.org/10.1007/ s10712-017-9442-2.

Betts, A. K., 1985: Mixing line analysis of clouds and cloudy boundary layers. J. Atmos. Sci., 42, 2751-2763, https://doi.org/ 10.1175/1520-0469(1985)042<2751:MLAOCA > 2.0.CO;2.

— 2000: Idealized model for equilibrium boundary layer over land. J. Hydrometeor., 1, 507-523, https://doi.org/10.1175/ 1525-7541(2000)001<0507:IMFEBL>2.0.CO;2.

— , and W. Ridgway, 1989: Climatic equilibrium of the atmospheric convective boundary layer over a tropical ocean. 
J. Atmos. Sci., 46, 2621-2641, https://doi.org/10.1175/15200469(1989)046<2621:CEOTAC $>2.0 . C O ; 2$.

Bony, S., and Coauthors, 2017: EUREC ${ }^{4}$ A: A field campaign to elucidate the couplings between clouds, convection and circulation. Surv. Geophys., 38, 1529-1568, https://doi.org/ 10.1007/s10712-017-9428-0.

Bretherton, C. S., and P. N. Blossey, 2017: Understanding mesoscale aggregation of shallow cumulus convection using largeeddy simulation. J. Adv. Model. Earth Syst., 9, 2798-2821, https://doi.org/10.1002/2017MS000981.

—_ _ - and M. Khairoutdinov, 2005: An energy-balance analysis of deep convective self-aggregation above uniform SST. J. Atmos. Sci., 62, 4273-4292, https://doi.org/10.1175/ JAS3614.1.

Deardorff, J., G. Willis, and D. Lilly, 1974: Comment on the paper by A.K. Betts "Non-precipitating cumulus convection and its parameterization.” Quart. J. Roy. Meteor. Soc., 100, 122-123, https://doi.org/10.1002/qj.49710042311.

Emanuel, K. A., J. D. Neelin, and C. S. Bretherton, 1994: On large-scale circulations in convecting atmospheres. Quart. J. Roy. Meteor. Soc., 120,1111-1143, https://doi.org/10.1002/ qj.49712051902.

Fläschner, D., T. Mauritsen, B. Stevens, and S. Bony, 2018: The signature of shallow circulations, not cloud radiative effects, in the spatial distribution of tropical precipitation. J. Climate, 31, 9489-9505, https://doi.org/10.1175/JCLI-D-18-0230.1.

Klingebiel, M., and Coauthors, 2019: Remote sensing of sea salt aerosol below trade wind clouds. J. Atmos. Sci., 76, 1189-1202, https://doi.org/10.1175/JAS-D-18-0139.1.

Klocke, D., M. Brsueck, C. Hohenegger, and B. Stevens, 2017: Rediscovering the doldrums in cloud resolving simulations of the tropical Atlantic. Nat. Geosci., 10, 891-896, https://doi.org/ 10.1038/s41561-017-0005-4.

Lareau, N. P., Y. Zhang, and S. A. Klein, 2018: Observed boundary layer controls on shallow cumulus at the ARM Southern Great Plains site. J. Atmos. Sci., 75, 2235-2255, https://doi.org/ 10.1175/JAS-D-17-0244.1.

Lilly, D. K., 1968: Models of cloud-topped mixed layers under a strong inversion. Quart. J. Roy. Meteor. Soc., 94, 292-309, https://doi.org/10.1002/qj.49709440106.

Lindzen, R. S., and S. Nigam, 1987: On the role of sea surface temperature gradients in forcing low-level winds and convergence in the tropics. J. Atmos. Sci., 44, 2418-2436, https://doi.org/10.1175/1520-0469(1987)044<2418:OTROSS > 2.0.CO;2.

Lonitz, K., B. Stevens, L. Nuijens, V. Sant, L. Hirsch, and A. Seifert, 2015: The signature of aerosols and meteorology in long-term cloud radar observations of trade wind cumuli. J. Atmos. Sci., 72, 4643-4659, https://doi.org/10.1175/JAS-D-14-0348.1.

Mapes, B. E., and P. Zuidema, 1996: Radiative-dynamical consequences of dry tongues in the tropical troposphere. J. Atmos. Sci., 53, 620-638, https://doi.org/10.1175/1520-0469(1996) $053<0620$ :RDCODT $>2.0 . \mathrm{CO} ; 2$.

Muller, C. J., and I. M. Held, 2012: Detailed investigation of the self-aggregation of convection in cloud-resolving simulations. J. Atmos. Sci., 69, 2551-2565, https://doi.org/10.1175/ JAS-D-11-0257.1.

Naumann, A. K., B. Stevens, C. Hohenegger, and J. P. Mellado, 2017: A conceptual model of a shallow circulation induced by prescribed low-level radiative cooling. J. Atmos. Sci., 74, 3129-3144, https://doi.org/10.1175/JAS-D-17-0030.1.

Neggers, R., B. Stevens, and J. D. Neelin, 2006: A simple equilibrium model for shallow-cumulus-topped mixed layers.
Theor. Comput. Fluid Dyn., 20, 305-322, https://doi.org/10.1007/ s00162-006-0030-1.

Nicholls, M. E., R. A. Pielke, and W. R. Cotton, 1991: Thermally forced gravity waves in an atmosphere at rest. J. Atmos. Sci., 48, 1869-1884, https://doi.org/10.1175/1520-0469(1991) 048<1869:TFGWIA > 2.0.CO;2.

Nigam, S., 1997: The annual warm to cold phase transition in the eastern equatorial Pacific: Diagnosis of the role of stratus cloud-top cooling. J. Climate, 10, 2447-2467, https://doi.org/ 10.1175/1520-0442(1997)010<2447:TAWTCP >2.0.CO;2.

Nilsson, J., and K. A. Emanuel, 1999: Equilibrium atmospheres of a two-column radiative-convective model. Quart. J. Roy. Meteor. Soc., 125, 2239-2264, https://doi.org/10.1002/qj.49712555814.

Nishant, N., S. Sherwood, and O. Geoffroy, 2016: Radiative driving of shallow return flows from the ITCZ. J. Adv. Model. Earth Syst., 8, 831-842, https://doi.org/10.1002/2015MS000606.

Nolan, D. S., C. Zhang, and S.-h. Chen, 2007: Dynamics of the shallow meridional circulation around intertropical convergence zones. J. Atmos. Sci., 64, 2262-2285, https://doi.org/ 10.1175/JAS3964.1.

Nuijens, L., I. Serikov, L. Hirsch, K. Lonitz, and B. Stevens, 2014: The distribution and variability of low-level cloud in the North Atlantic trades. Quart. J. Roy. Meteor. Soc., 140, 2364-2374, https://doi.org/10.1002/qj.2307.

Rauber, R. M., and Coauthors, 2007: Rain in shallow cumulus over the ocean: The RICO campaign. Bull. Amer. Meteor. Soc., 88, 1912-1924, https://doi.org/10.1175/BAMS-88-12-1912.

Reynolds, R. W., T. M. Smith, C. Liu, D. B. Chelton, K. S. Casey, and M. G. Schlax, 2007: Daily high-resolution-blended analyses for sea surface temperature. J. Climate, 20, 5473-5496, https://doi.org/10.1175/2007JCLI1824.1.

Riehl, H., T. Yeh, J. S. Malkus, and N. E. La Seur, 1951: The northeast trade of the Pacific Ocean. Quart. J. Roy. Meteor. Soc., 77, 598-626, https://doi.org/10.1002/qj.49707733405.

Sarachik, E., 1978: Tropical sea surface temperature: An interactive one-dimensional atmosphere-ocean model. Dyn. Atmos. Oceans, 2, 455-469, https://doi.org/10.1016/0377-0265(78) 90014-3.

Schulz, H., and B. Stevens, 2018: Observing the tropical atmosphere in moisture space. J. Atmos. Sci., 75, 3313-3330, https:// doi.org/10.1175/JAS-D-17-0375.1.

Seifert, A., T. Heus, R. Pincus, and B. Stevens, 2015: Large-eddy simulation of the transient and near-equilibrium behavior of precipitating shallow convection. J. Adv. Model. Earth Syst., 7, 1918-1937, https://doi.org/10.1002/2015MS000489.

Sobel, A. H., and C. S. Bretherton, 2000: Modeling tropical precipitation in a single column. J. Climate, 13, 4378-4392, https:// doi.org/10.1175/1520-0442(2000)013<4378:MTPIAS >2.0.CO;2.

— , and J. D. Neelin, 2006: The boundary layer contribution to intertropical convergence zones in the quasi-equilibrium tropical circulation model framework. Theor. Comput. Fluid Dyn., 20, 323-350, https://doi.org/10.1007/s00162-006-0033-y.

Stevens, B., 2006: Bulk boundary-layer concepts for simplified models of tropical dynamics. Theor. Comput. Fluid Dyn., 20, 279-304, https://doi.org/10.1007/s00162-006-0032-z.

— , and Coauthors, 2001: Simulations of trade wind cumuli under a strong inversion. J. Atmos. Sci., 58, 1870-1891, https:// doi.org/10.1175/1520-0469(2001)058<1870:SOTWCU $>$ 2.0. $\mathrm{CO} ; 2$

, and Coauthors, 2016: The Barbados Cloud Observatory: Anchoring investigations of clouds and circulation on the edge of the ITCZ. Bull. Amer. Meteor. Soc., 97, 787-801, https://doi.org/10.1175/BAMS-D-14-00247.1. 
H. Brogniez, C. Kiemle, J.-L. Lacour, C. Crevoisier, and J. Kiliani, 2017: Structure and dynamical influence of water vapor in the lower tropical troposphere. Surv. Geophys., 38 , 1371-1397, https://doi.org/10.1007/s10712-017-9420-8.

Tennekes, H., 1973: A model for the dynamics of the inversion above a convective boundary layer. J. Atmos. Sci., 30, 558-567, https://doi.org/10.1175/1520-0469(1973)030<0558:AMFTDO> 2.0.CO;2.

Trenberth, K. E., D. P. Stepaniak, and J. M. Caron, 2000: The global monsoon as seen through the divergent atmospheric circulation. J. Climate, 13, 3969-3993, https://doi.org/10.1175/ 1520-0442(2000)013<3969:TGMAST>2.0.CO;2.

Wang, Y., S.-P. Xie, B. Wang, and H. Xu, 2005: Large-scale atmospheric forcing by southeast Pacific boundary layer clouds: A regional model study. J. Climate, 18, 934-951, https:// doi.org/10.1175/JCLI3302.1.
Wing, A. A., and K. A. Emanuel, 2013: Physical mechanisms controlling self-aggregation of convection in idealized numerical modeling simulations. J. Adv. Model. Earth Syst., 6, 59-74, https://doi.org/10.1002/2013MS000269.

Wu, Z., 2003: A shallow CISK, deep equilibrium mechanism for the interaction between large-scale convection and large-scale circulations in the tropics. J. Atmos. Sci., 60, 377-392, https:// doi.org/10.1175/1520-0469(2003)060<0377:ASCDEM>2.0.CO;2. , D. S. Battisti, and E. Sarachik, 2000: Rayleigh friction, Newtonian cooling, and the linear response to steady tropical heating. J. Atmos. Sci., 57, 1937-1957, https://doi.org/10.1175/ 1520-0469(2000)057<1937:RFNCAT>2.0.CO;2.

Zhang, C., D. S. Nolan, C. D. Thorncroft, and H. Nguyen, 2008: Shallow meridional circulations in the tropical atmosphere. J. Climate, 21, 3453-3470, https://doi.org/10.1175/ 2007JCLI1870.1. 